\title{
Group A
}

[128]

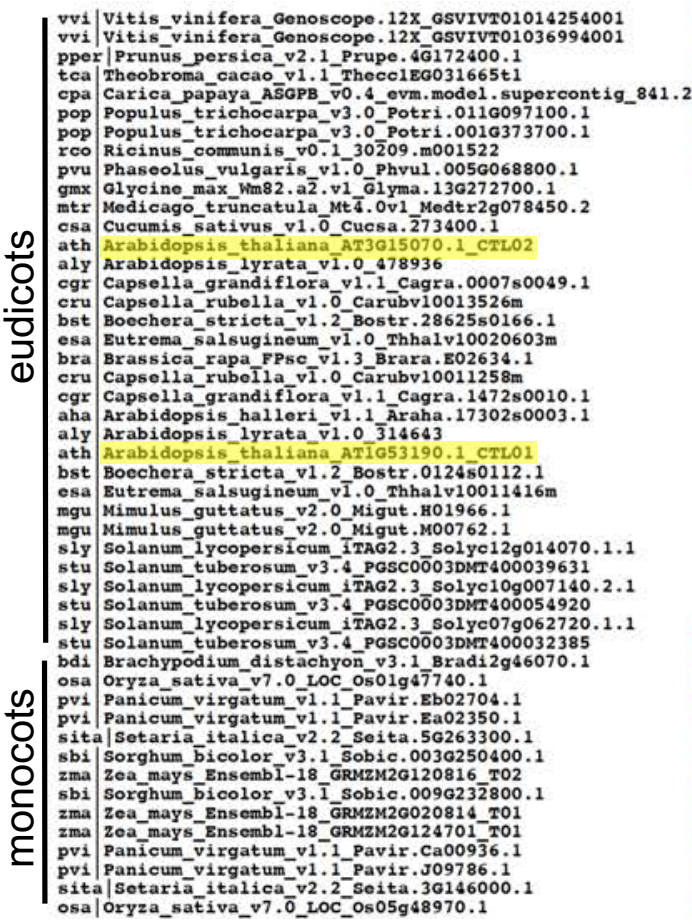

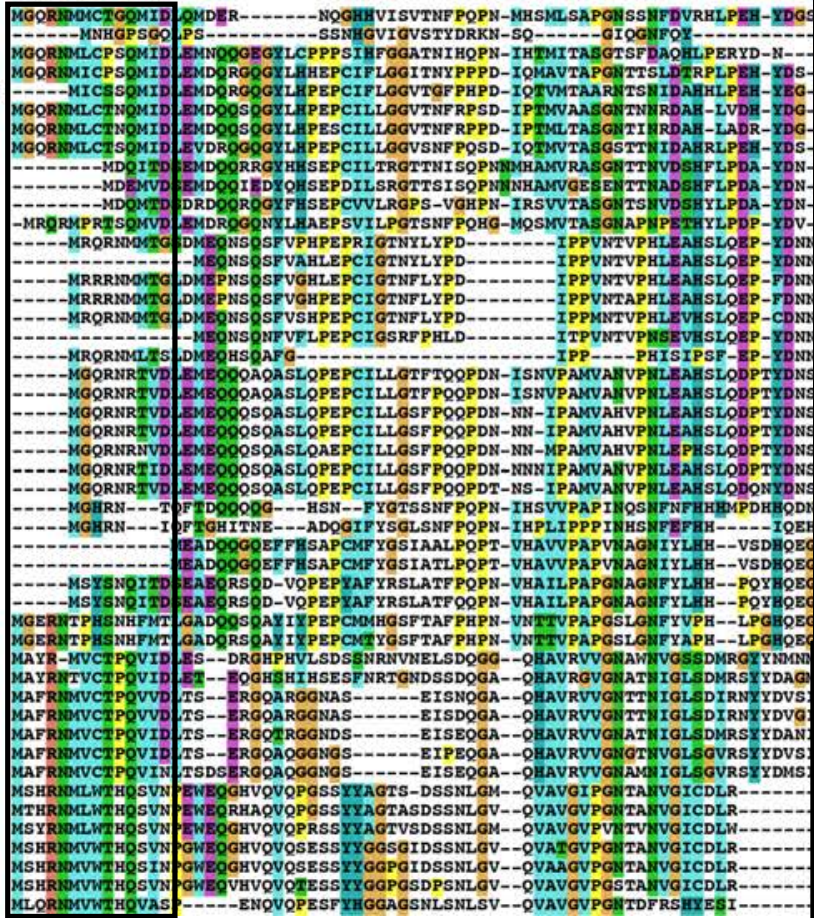

[129]

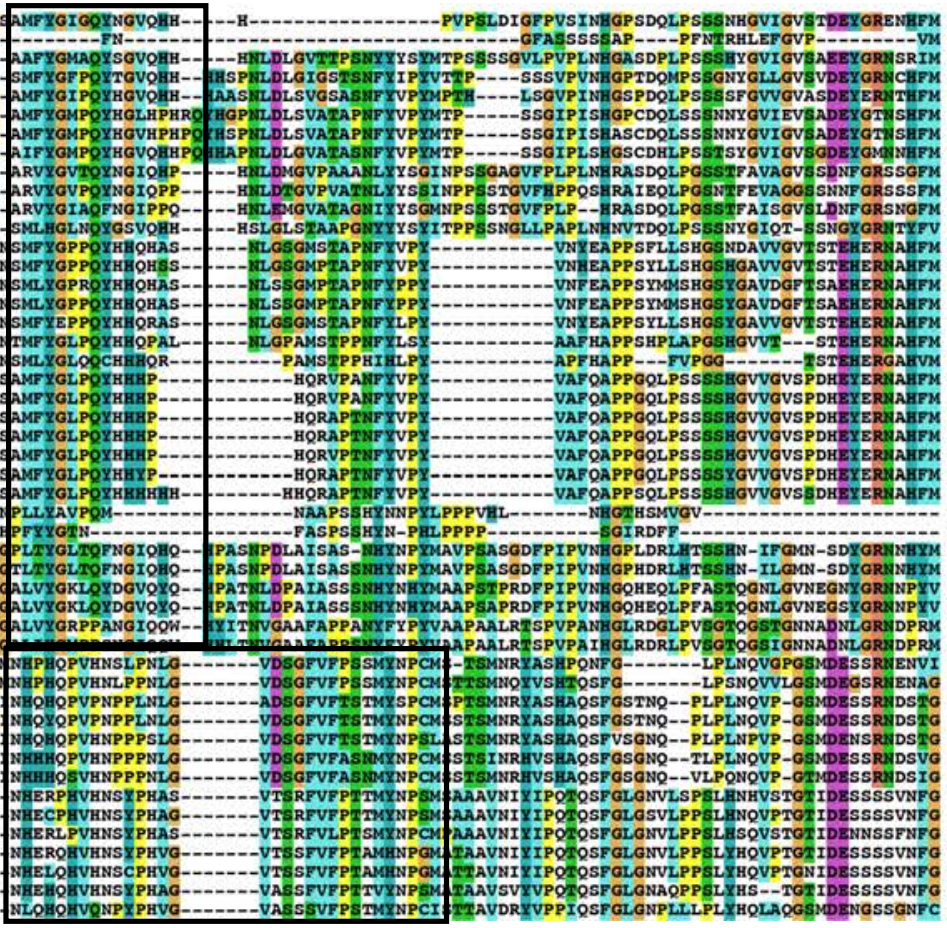

[147] 


\section{Group A (cont.)}

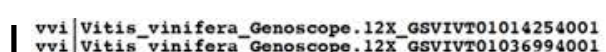

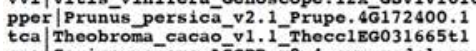

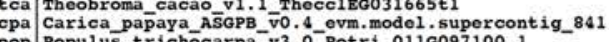
pop Populus_trichocarpa- ${ }^{3} 3.0$. Potri. 0016373700.

rco Ricinus_conmunis_v0.1_- 30209 .mo01522

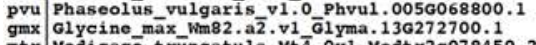

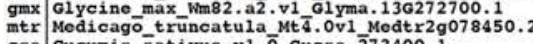

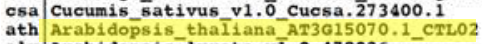
egr Capsella_grandif1ora_vi.1_cagra.0007s0049.1

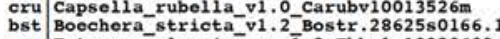

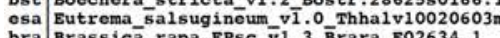

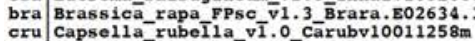

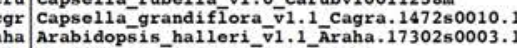

1y Axabidopsis_- $y$ yrata_vi.0.

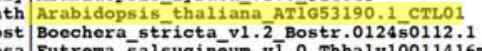

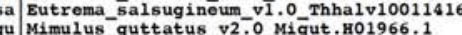

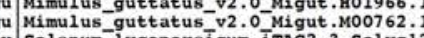

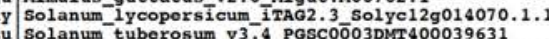

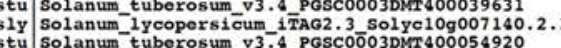

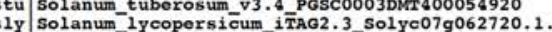

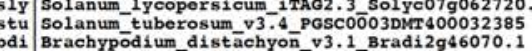

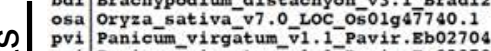

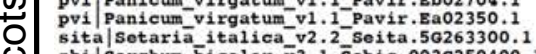

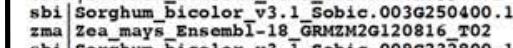

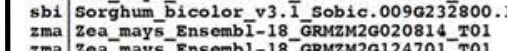

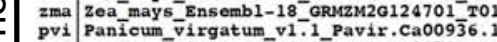

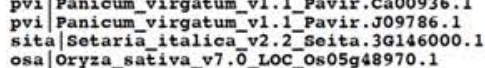
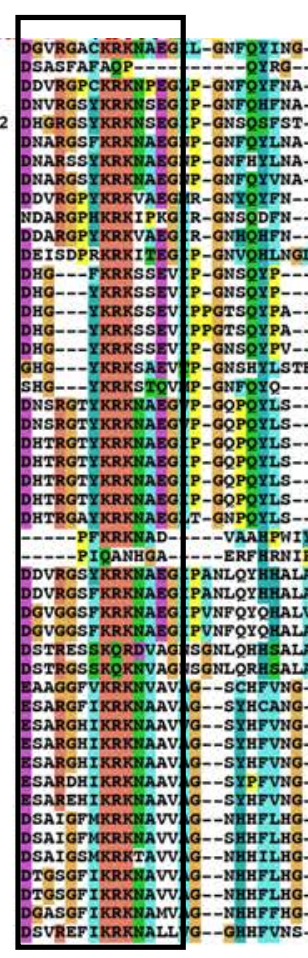

--LASSRNARAP

$$
\text { - SATSSISVPPSI }
$$

PSNIRNPDG-VAVMDAVTFALPOYVGTGNLPIMEAGPQSSMRNRSATTGLDSAMT-

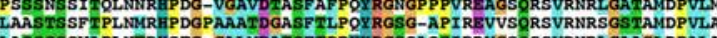

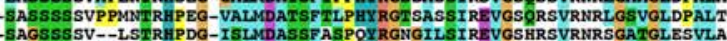

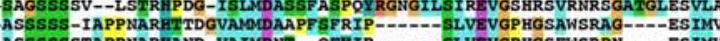
ASTSSS-TAPPNARHADG-VATMNT--QPFHT PCSFPOLNTSETAPFSFPHFC

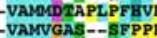
SLVGVGPHGGAWSRSG----ESTMV -

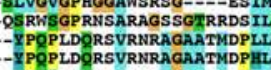
\begin{tabular}{l} 
YPOVLDRSVRNRAGAATMDPLLS \\
YPOVLDRSVRNRAGAMTDPLIS \\
YPOPLDORSVRNRAGATTIDPLLS \\
\hline
\end{tabular} PPQLMTPETAPISFPOFC TLAAPFNAPETIPPFC PQPLDQRSVWNRAGPATMDPCLS -TLAAPFNTPETIAPF -TLAAPFNTPETIAPY -TLAAPFMPERTIAPFC

$-\cdot-$
MAPMIARAHESDVPMDAAST SSVAPMIARAHESDVSMDARAS FPLA-

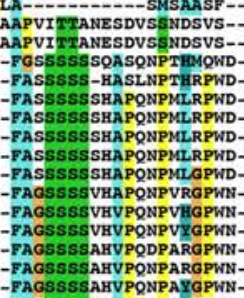

[107]

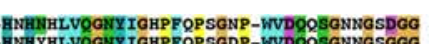

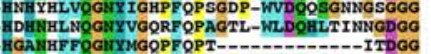

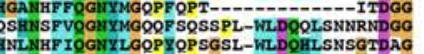

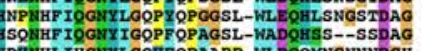

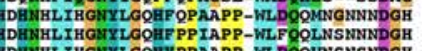
DDHHLIHGNYLOOHYPPAAPP-WLDQQVNSSHSNDGK WAFS SOEXYA-AHPFPPLGSTWXDOHCNGSRSDOS

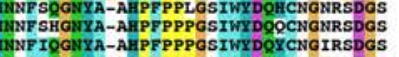

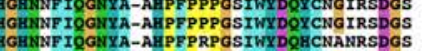
MUANFFIQGSYL-AHPFPPPGSVWXDOBCNGNTS AAPNFVOGSYAGH PFPPPGSVWYDQRHG--R MAPNNFI OGNXAGHHPFPPPGSIWXDOHHG--

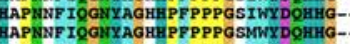
APRMPVOGMYAGHHPFPPPGSIWYD

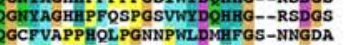
GPSSSVESDVALMDNSRVQYV EESFRKKNAEVAFTSYZLYQNG

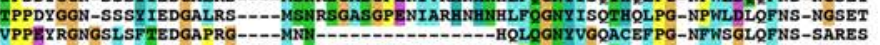

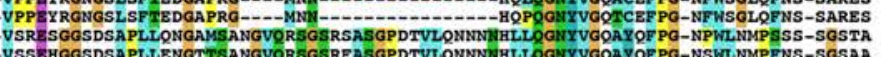

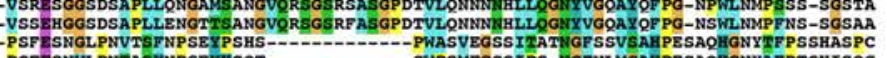

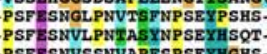
PSFESHVSSNVAPFPSEXHGHS
PSFESSVSSNVAPFNPSEYHGHS
PSFESNVSSNVAPFNPSEYHSRS SWPSMEGSS IPS-MGFNLMGALPESAQHGNYAFPTSHISQC

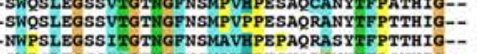

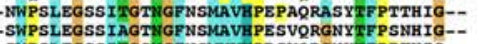
SWPSLEGSS ITGSHGFNSMVVIPDSVQRGNYIFP PTHIS-

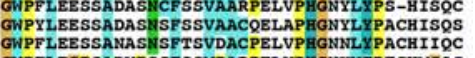
GWPFLEGPSADVPSSFSSMPARPELVPHGNYYFPTCHMGOC

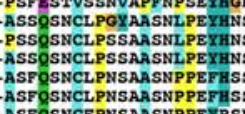

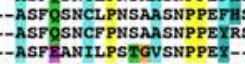

GWL FLEGSSADVPS -SSADSLINISNSMASIRPELVIRGNYVFPAGHMSOY 


\section{Group A (cont.)}
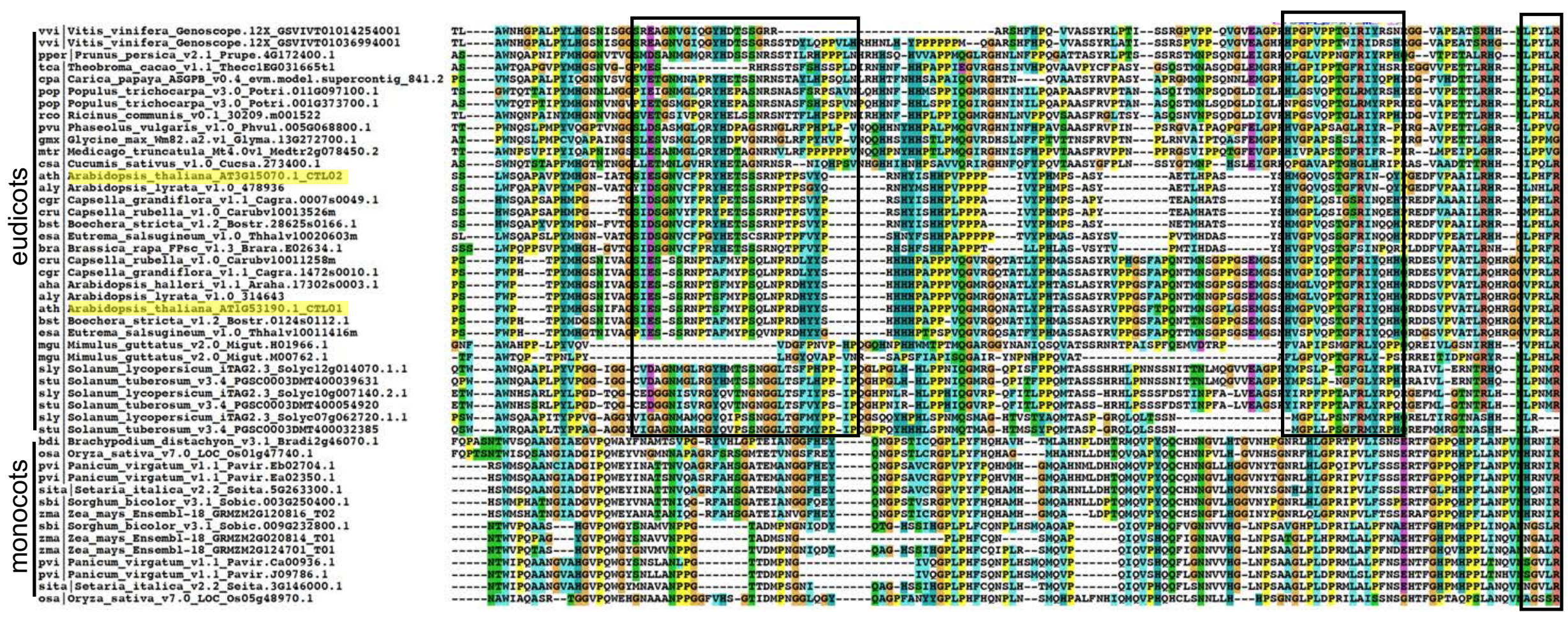

[140]

[135]

[130] 


\section{Group A (cont.)}

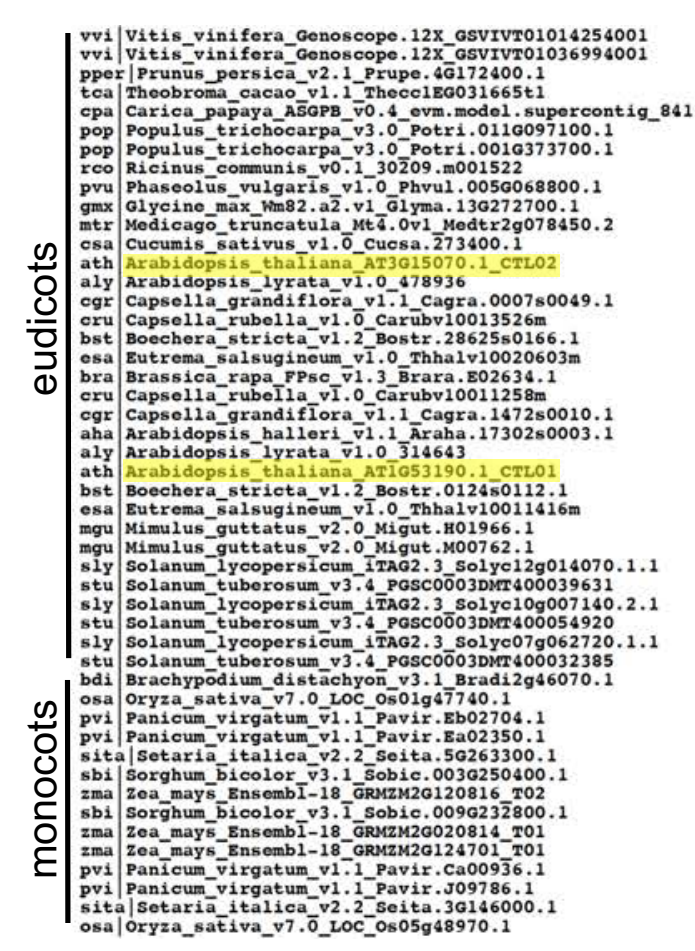

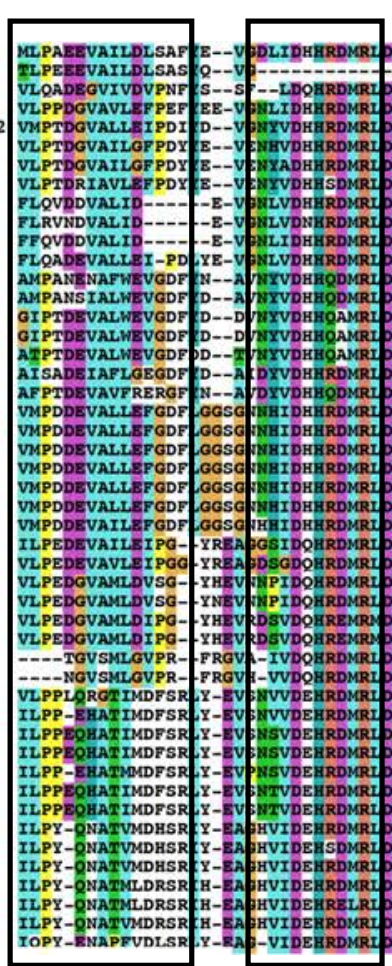

[130]
[102]

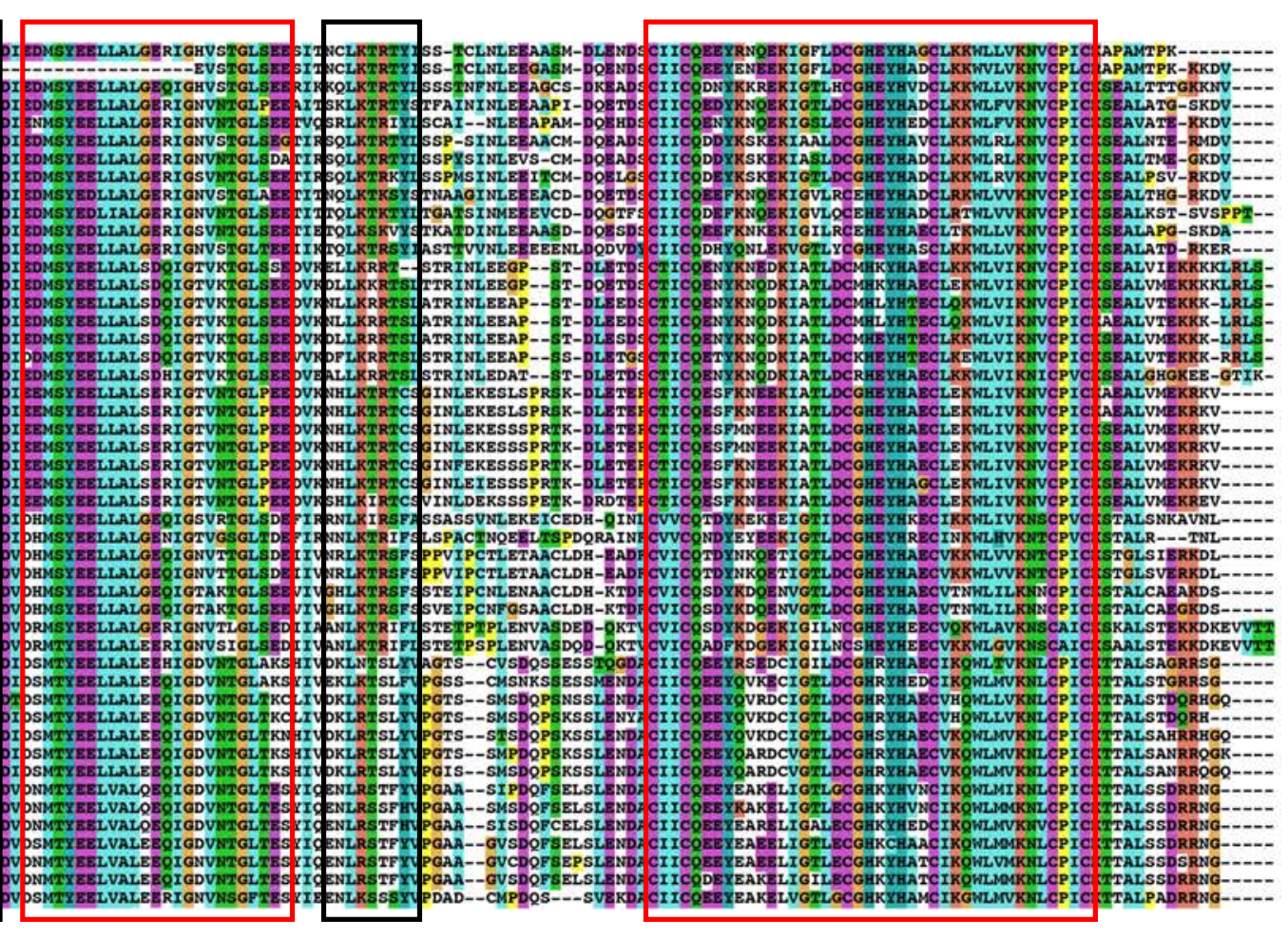

[136]

RING-H2 

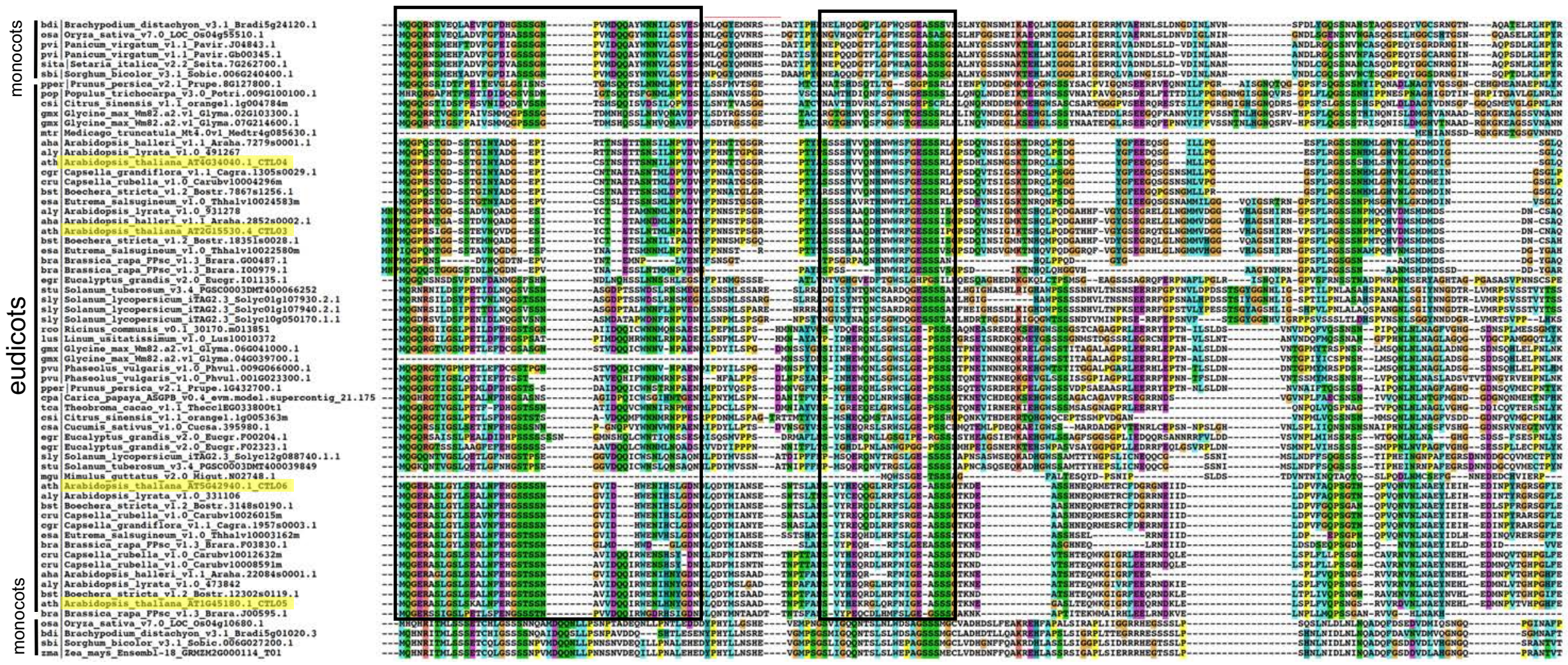

[111]
[120] 


\section{Group B (cont.)}

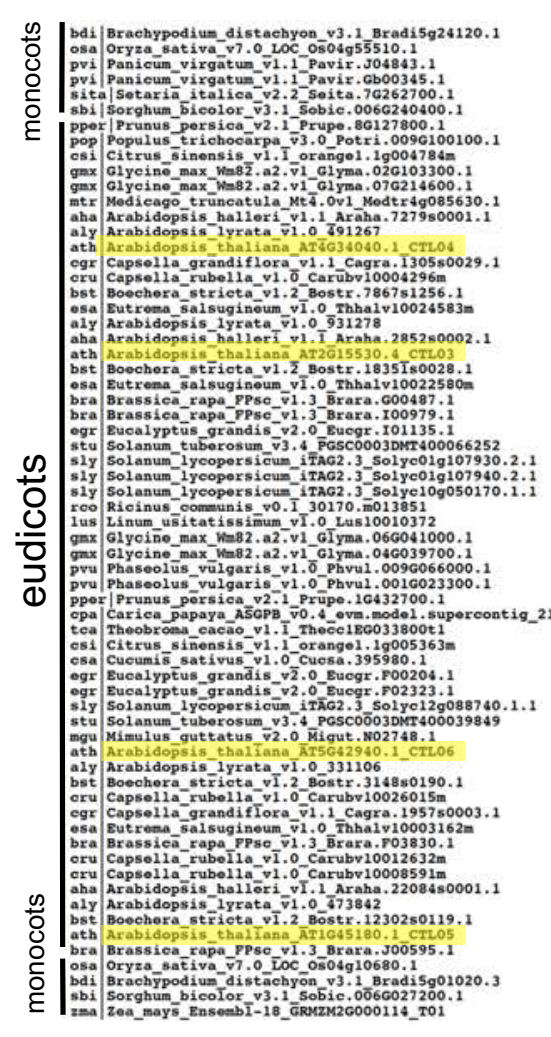

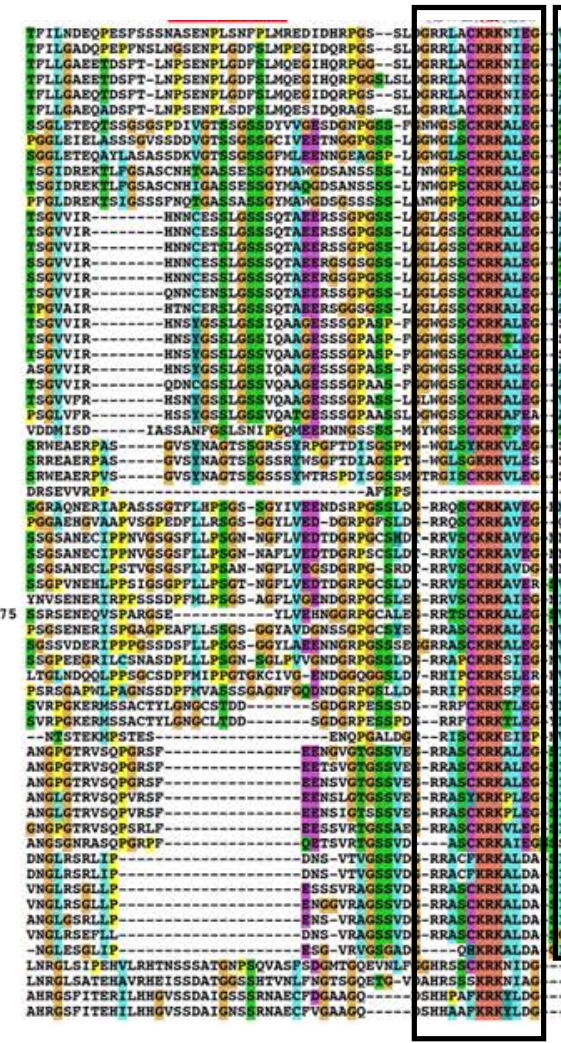

[107]

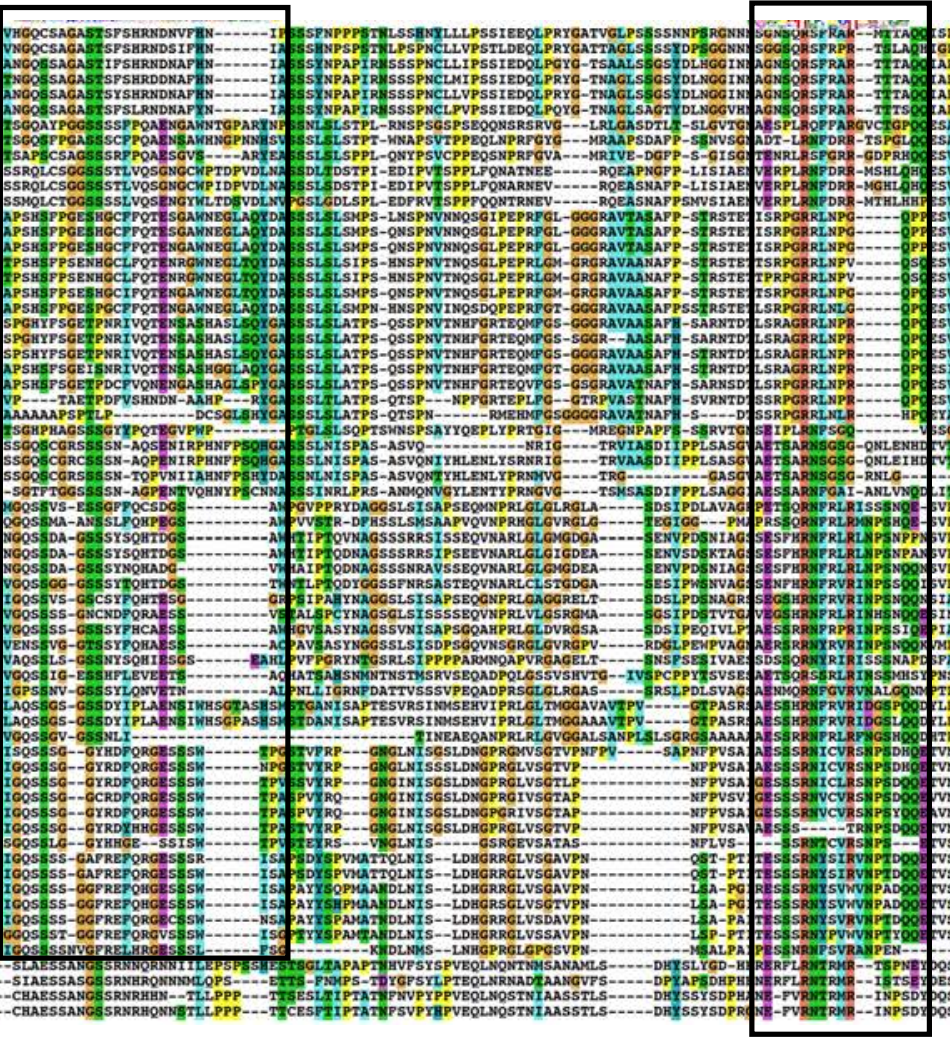

[116]

[139]

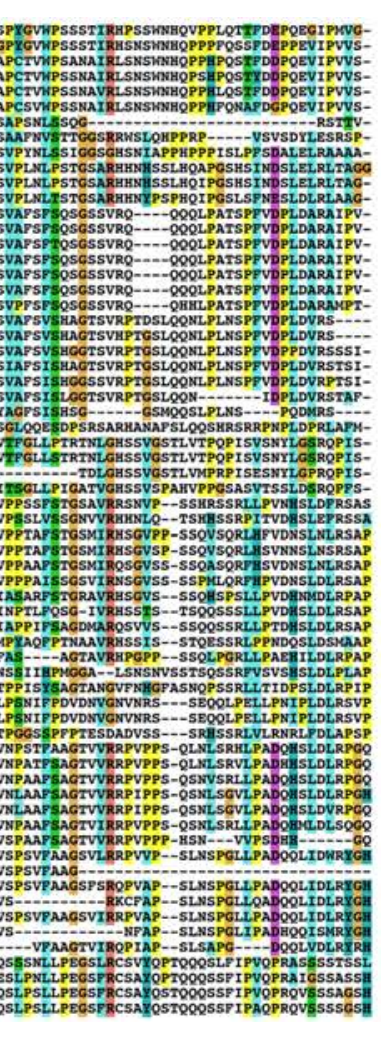




\section{Group B (cont.)}

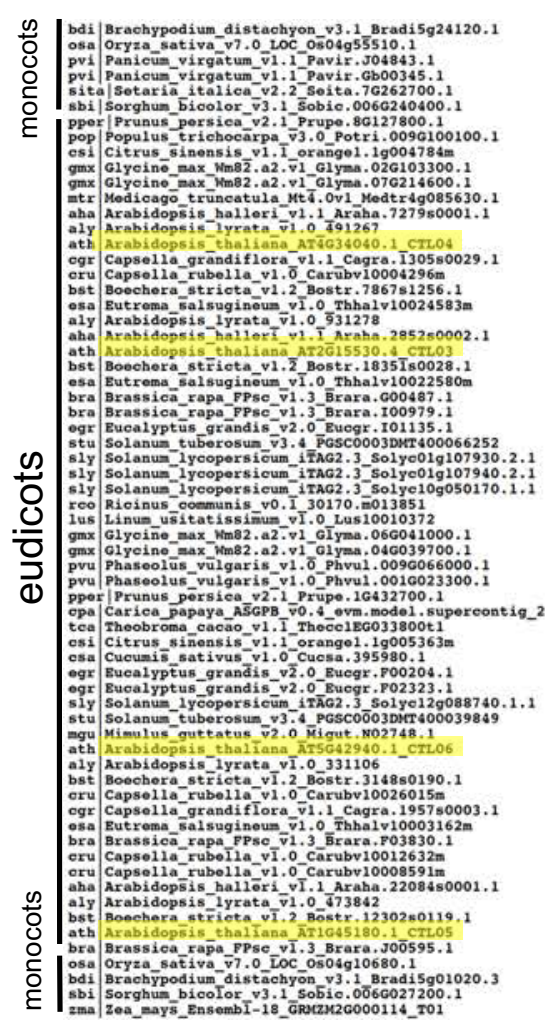

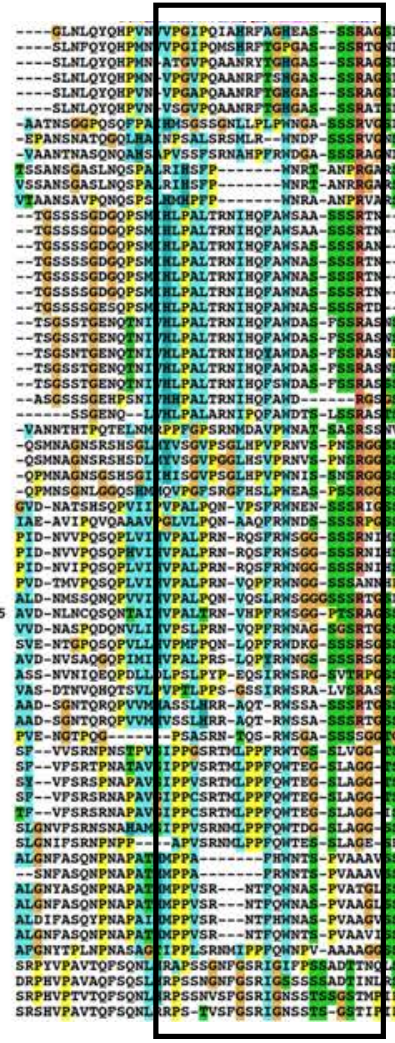

[114]

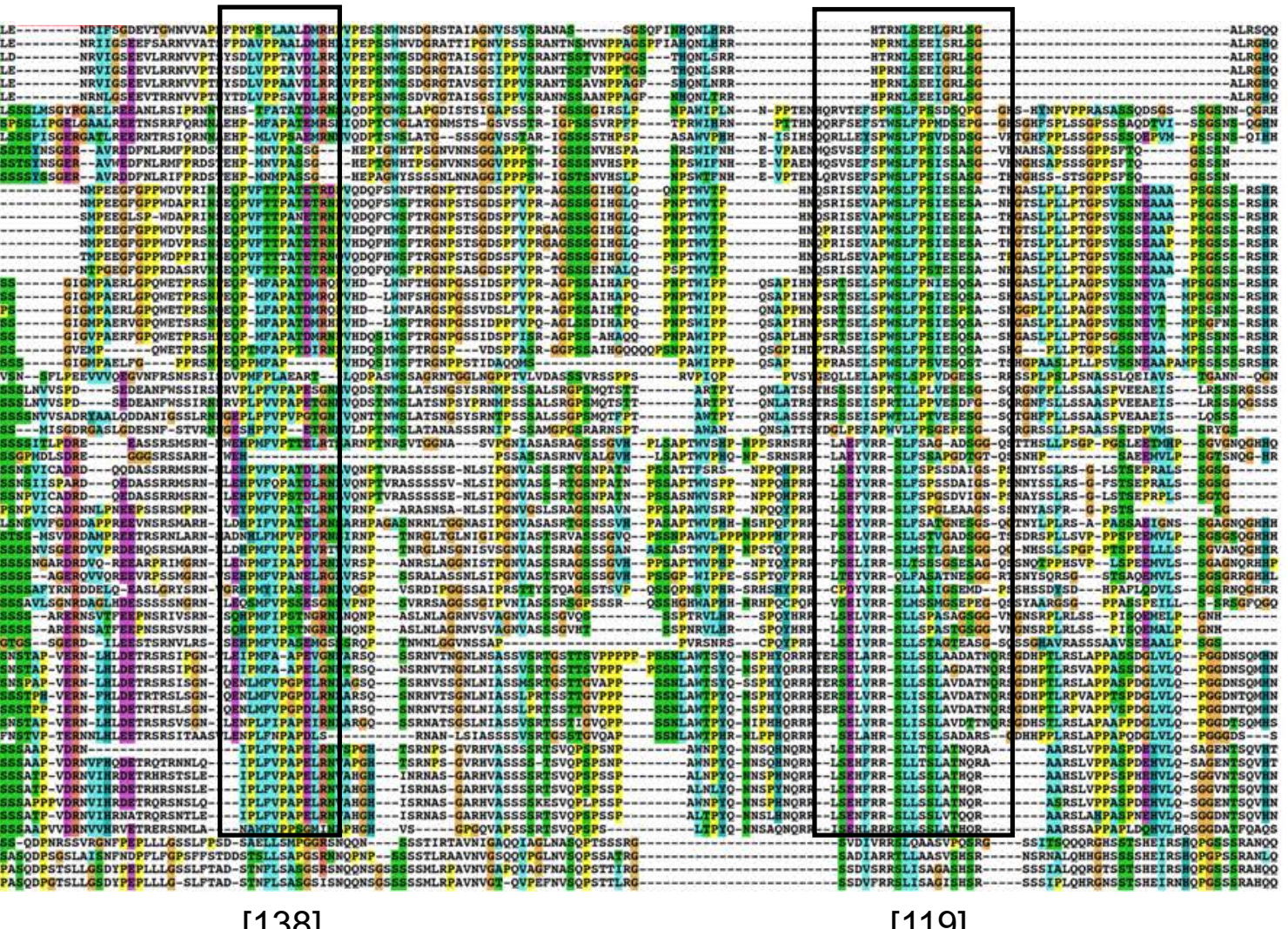

[138]

[119] 


\section{Group B (cont.)}
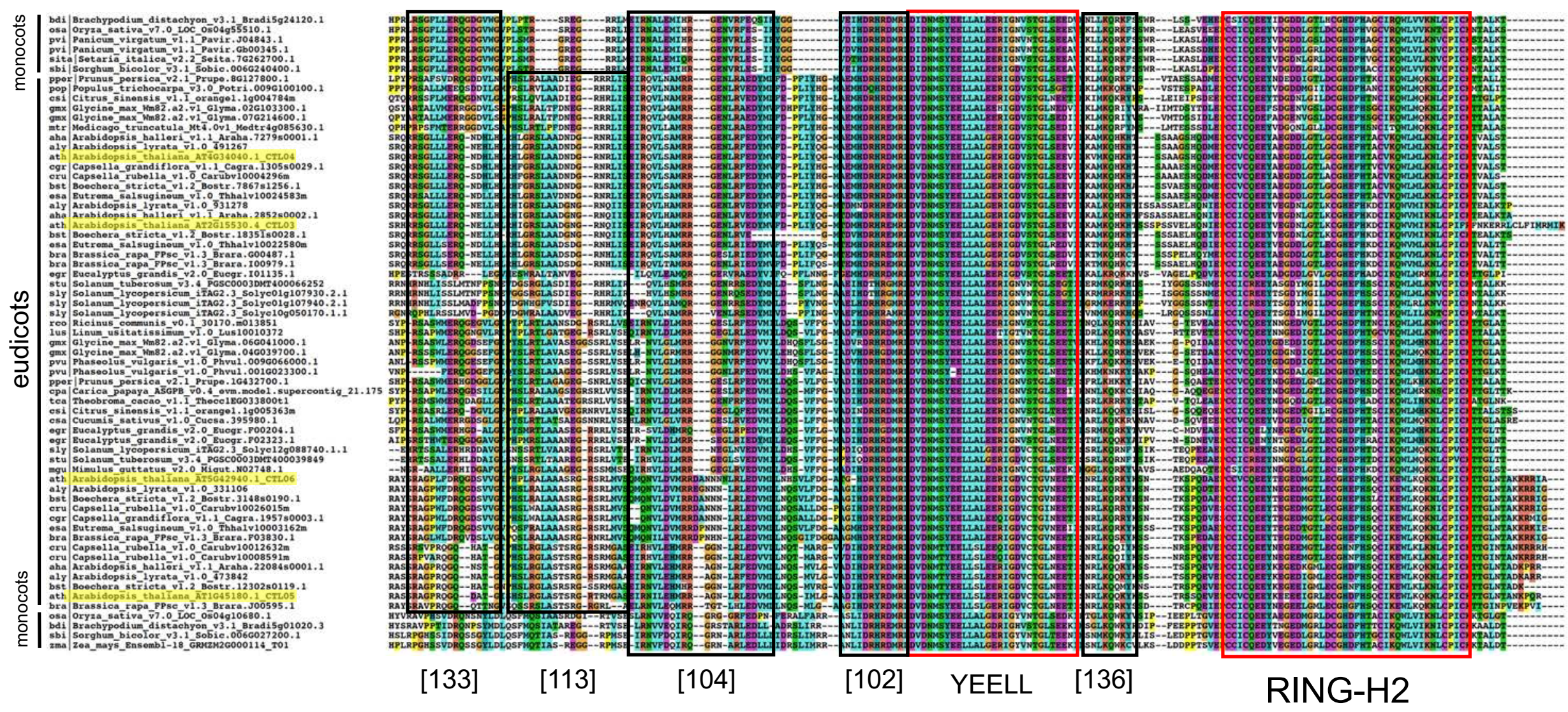


\section{Group C}

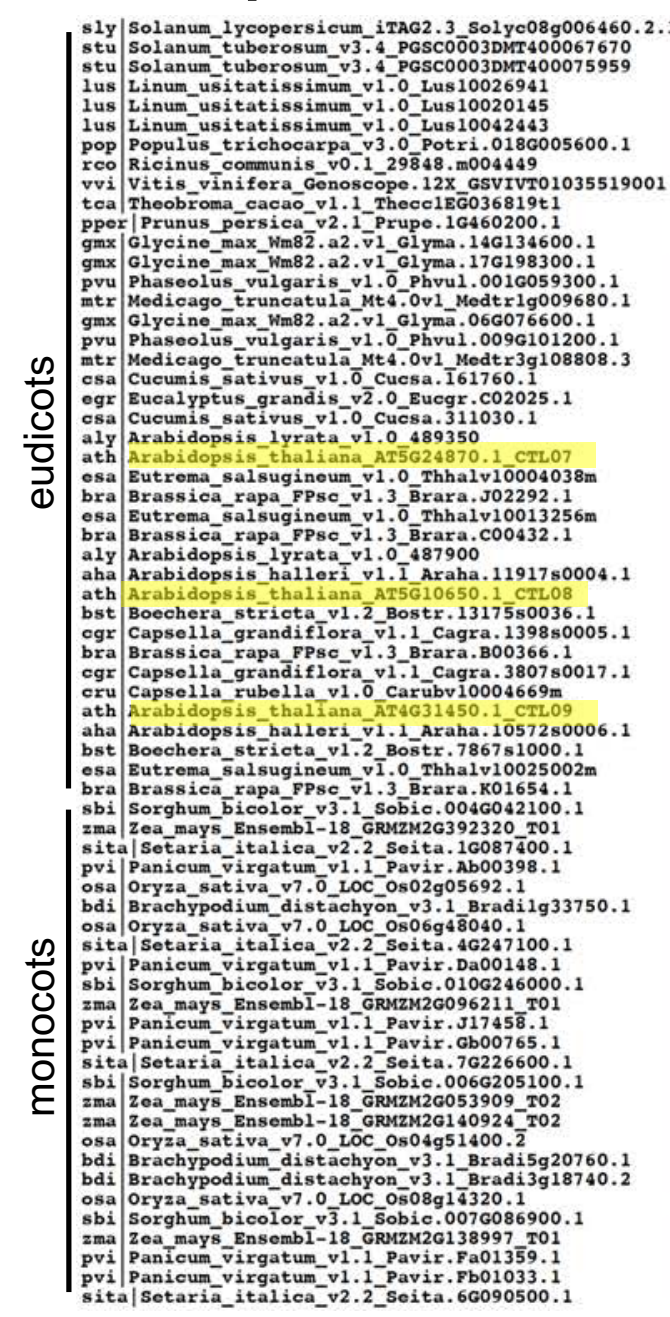

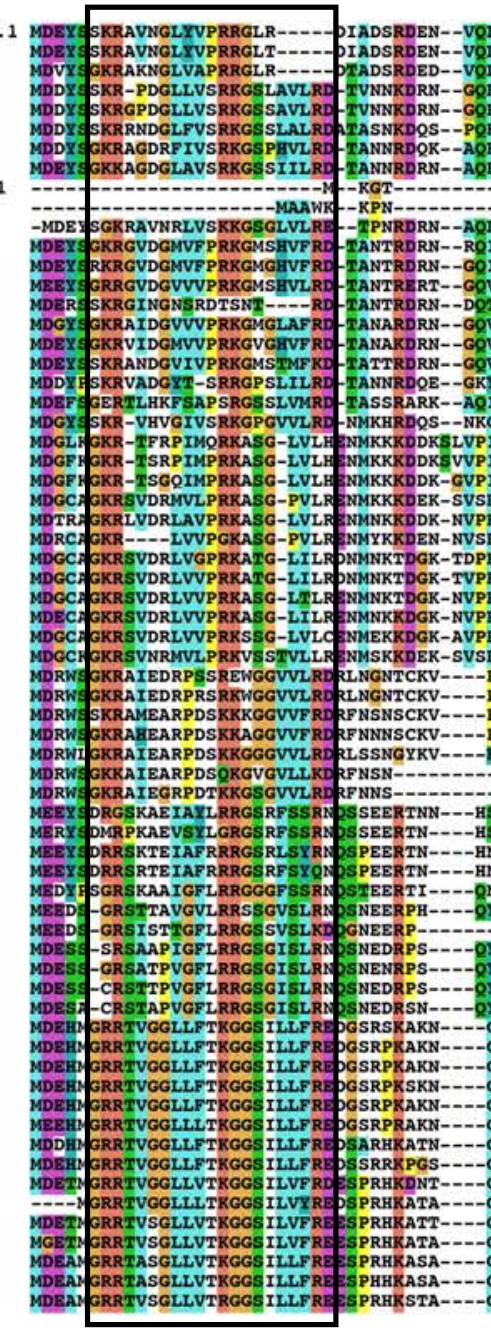

[126]
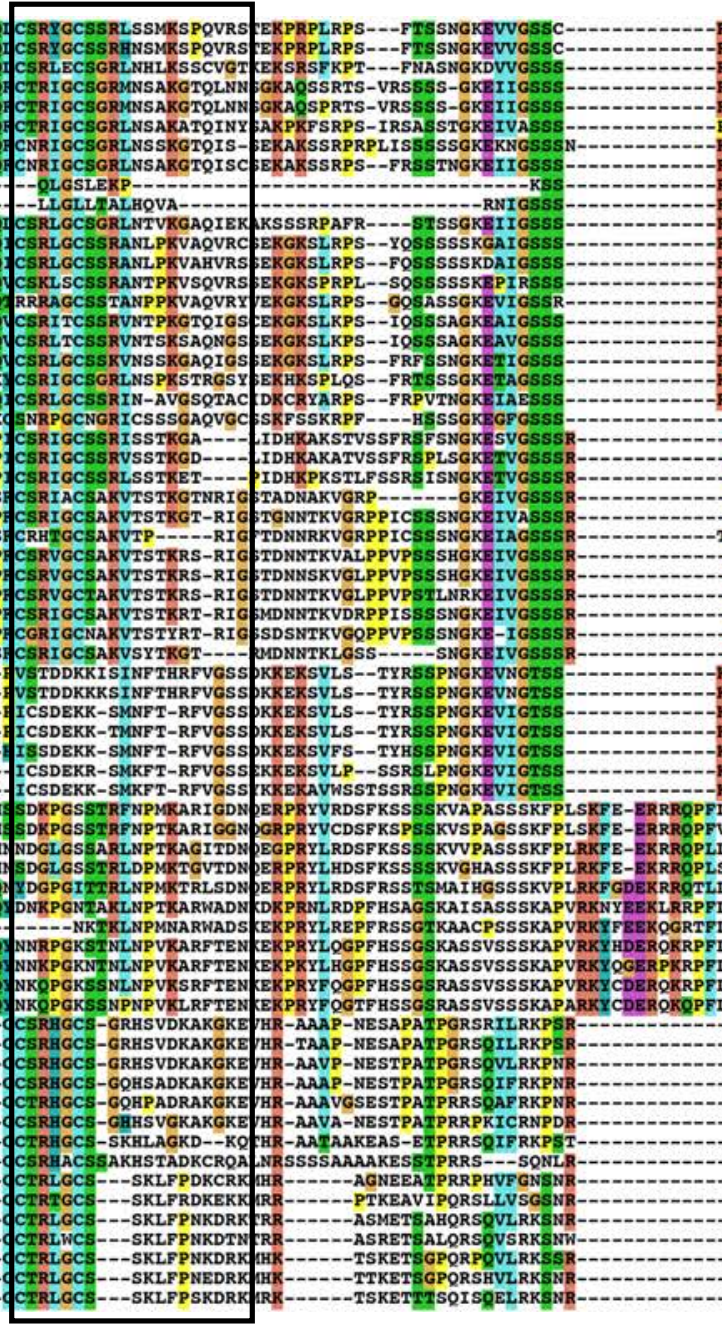

RTSSVMANARKSFKD---RKAYSHVGNDOSETSCSHGEPEASEASERMKLSKV
RTPSVMANARKSLRDD---RKTNSHVGNDOSETCSHGEP---EASEHKKSKKA

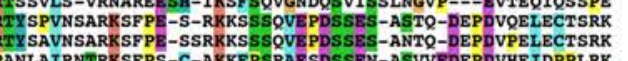

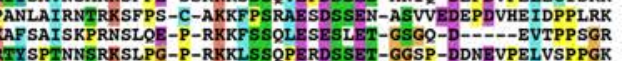

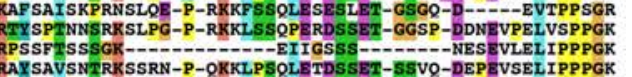

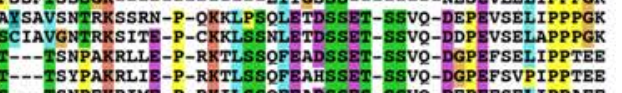

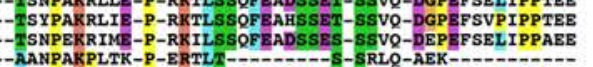

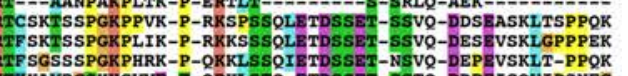

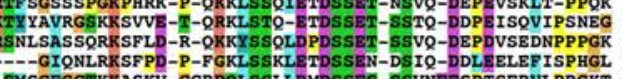

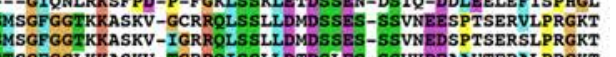

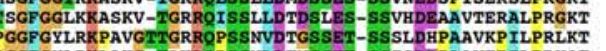

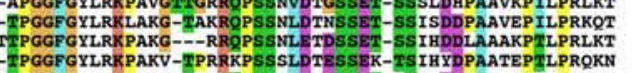

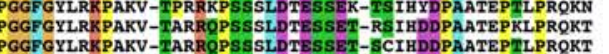

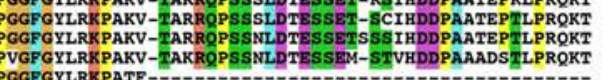

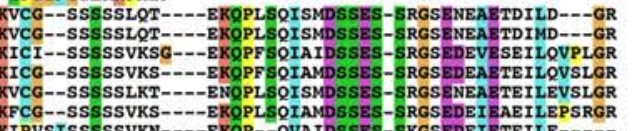

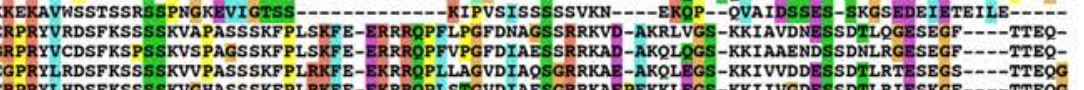

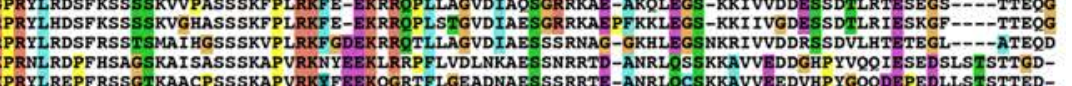

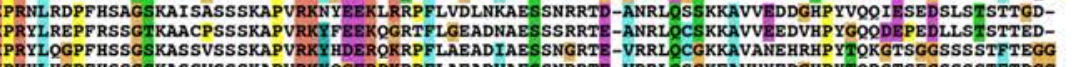

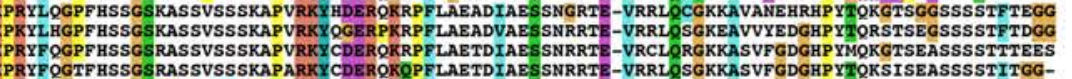

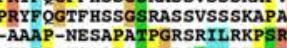

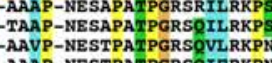

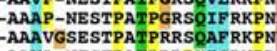

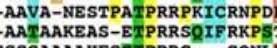

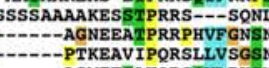

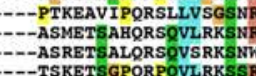

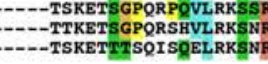

[117] 


\section{Group C (cont.)}

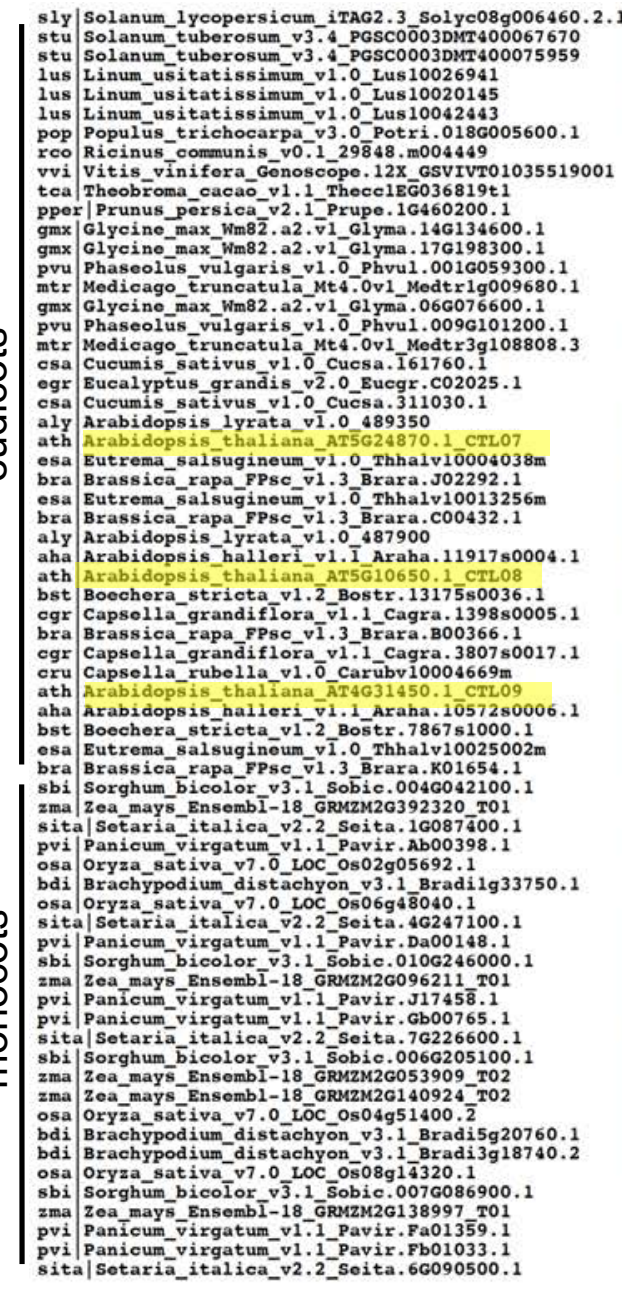

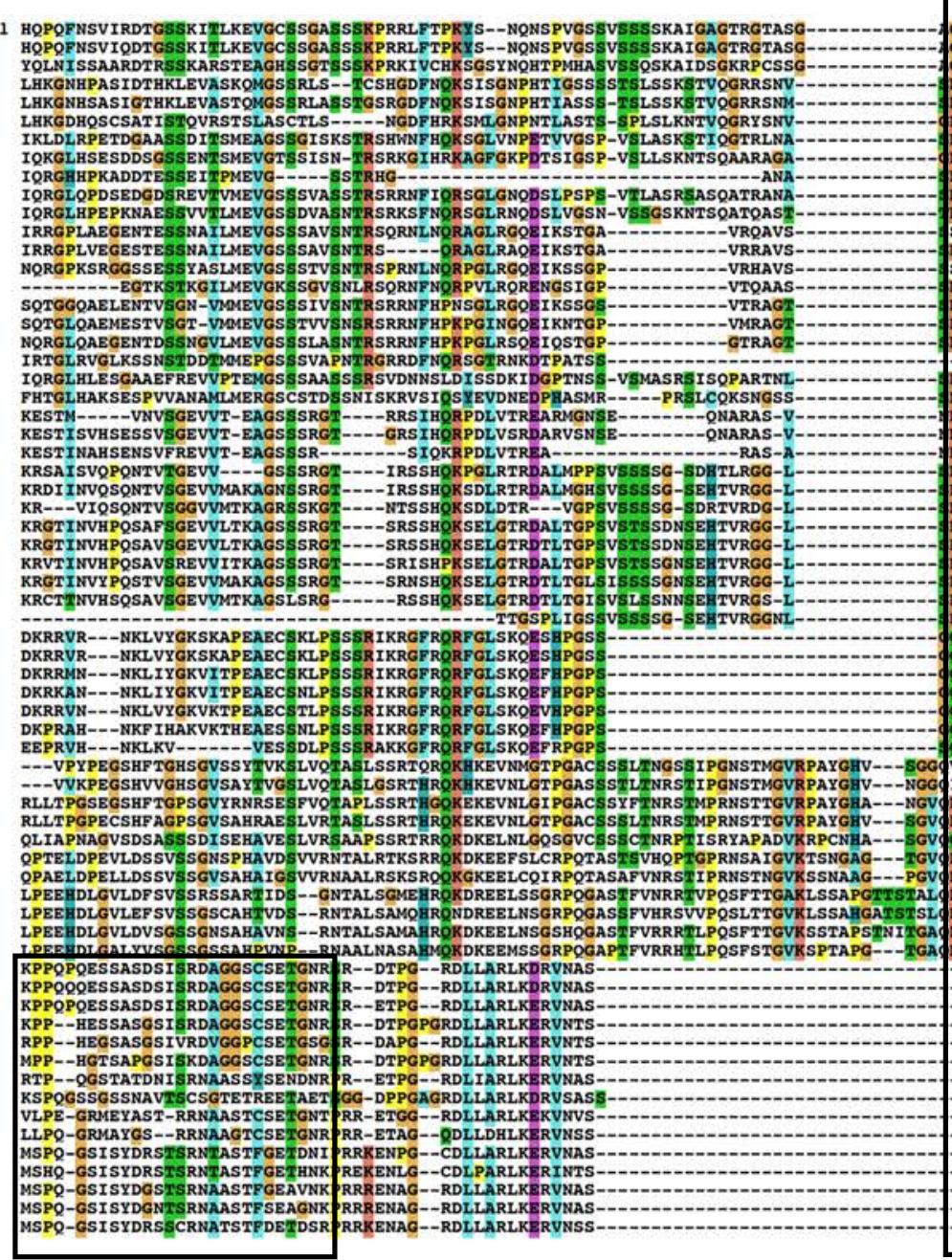

[148]

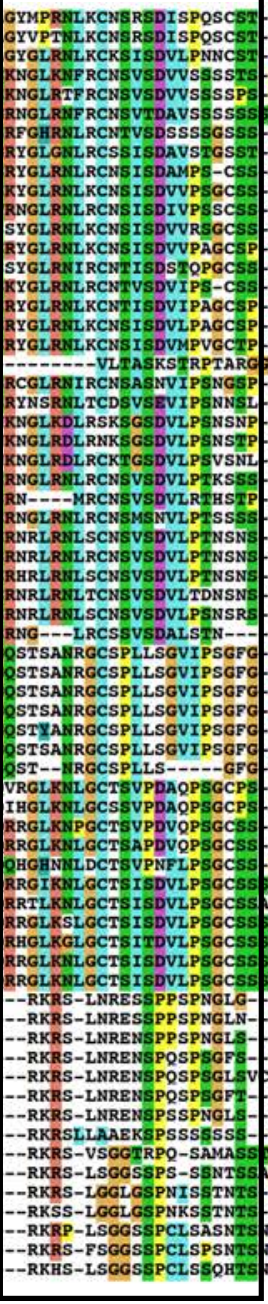

[108]

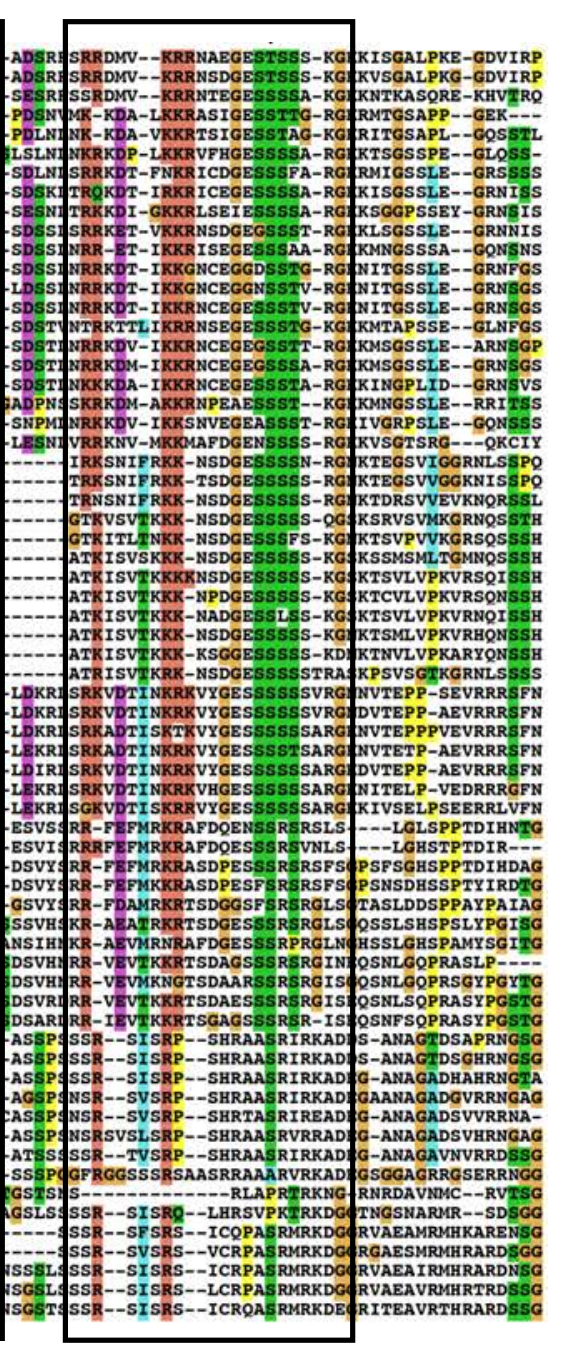

[122] 


\section{Group C (cont.)}
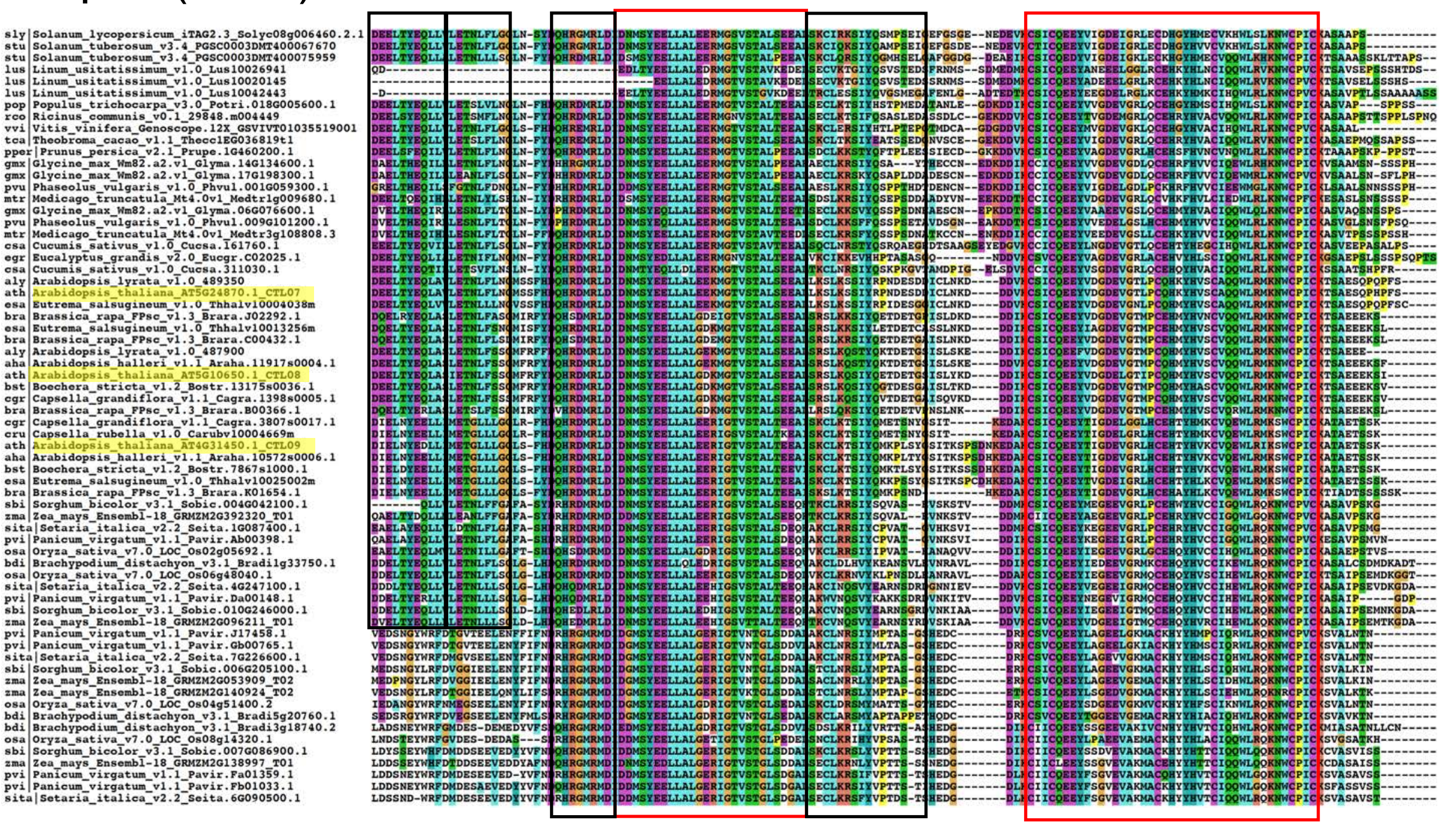

[103] [144] [102] YEELL [110]

RING-H2 


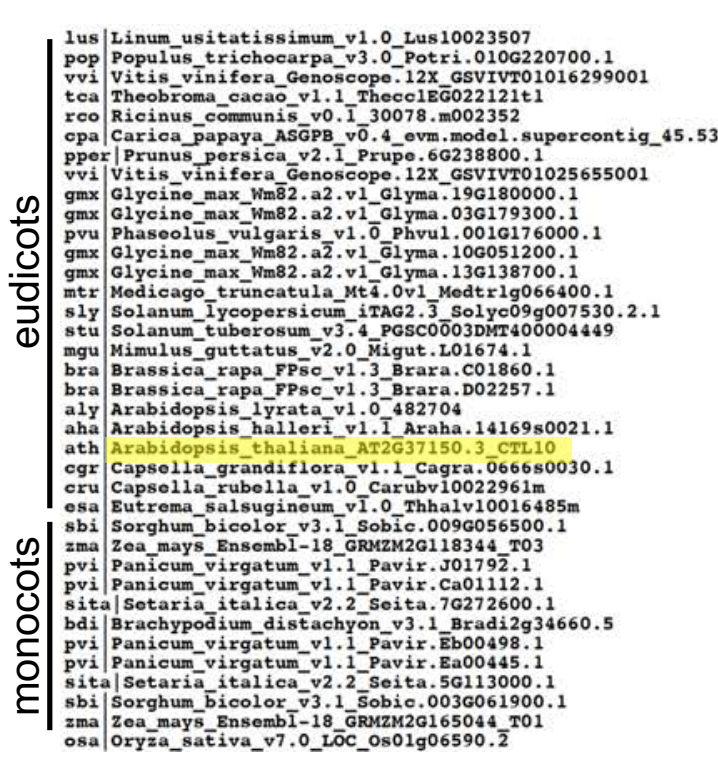

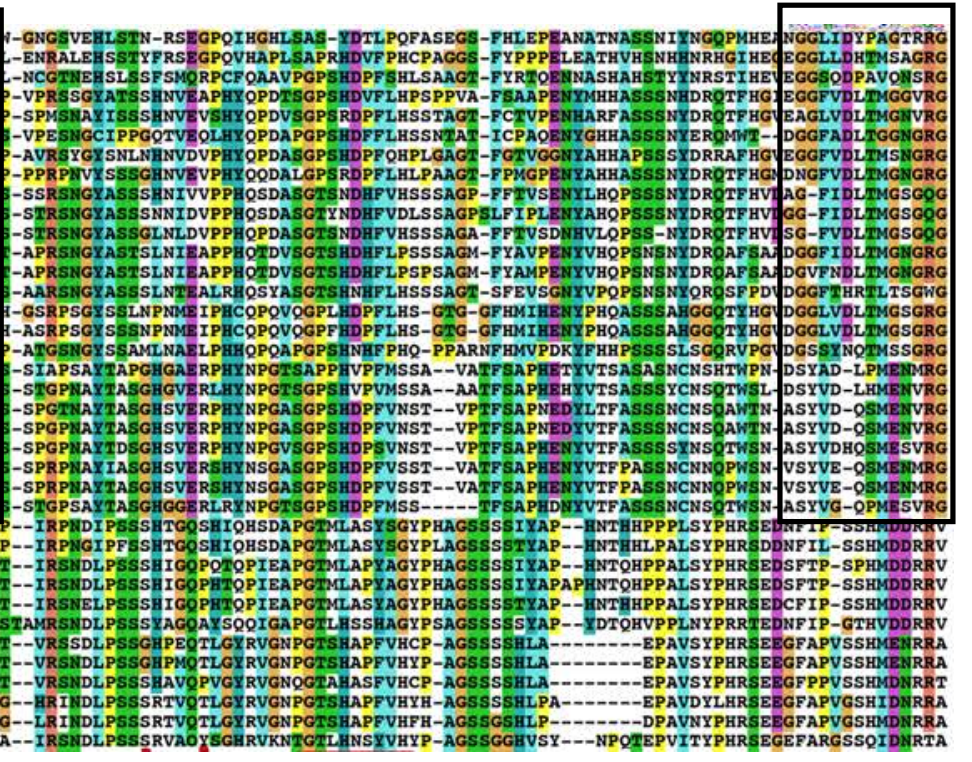

[129]

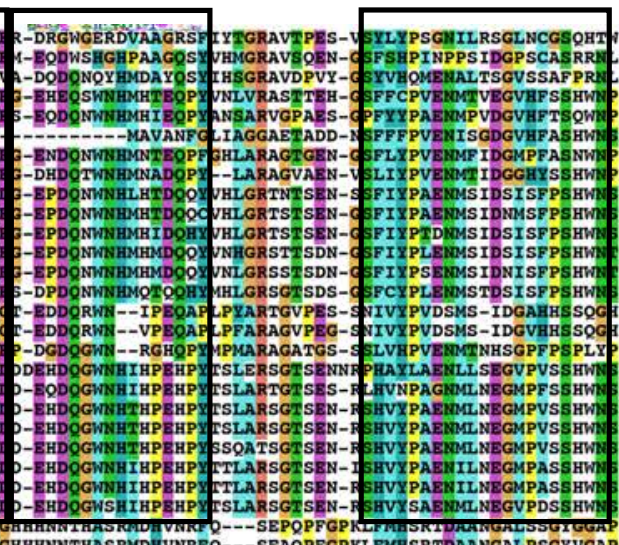

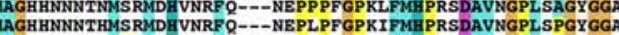
MAGHHHNNTHMPRMDQVNRF Q----NEPPPFGPKLFMI PRSDAAN---ASSYGGA

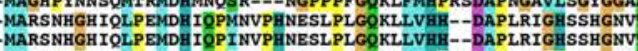

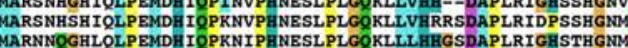

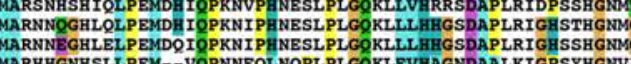
[146]
[145] 


\section{Group D (cont.)}

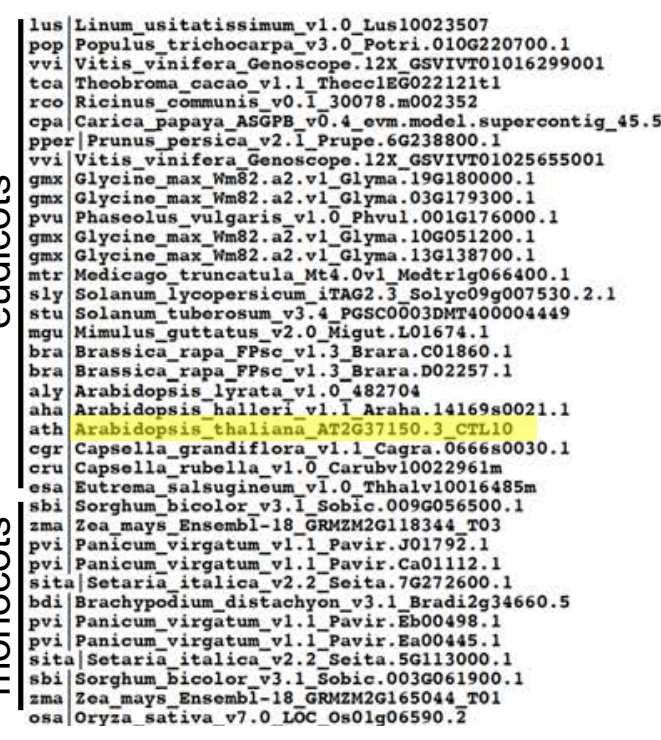

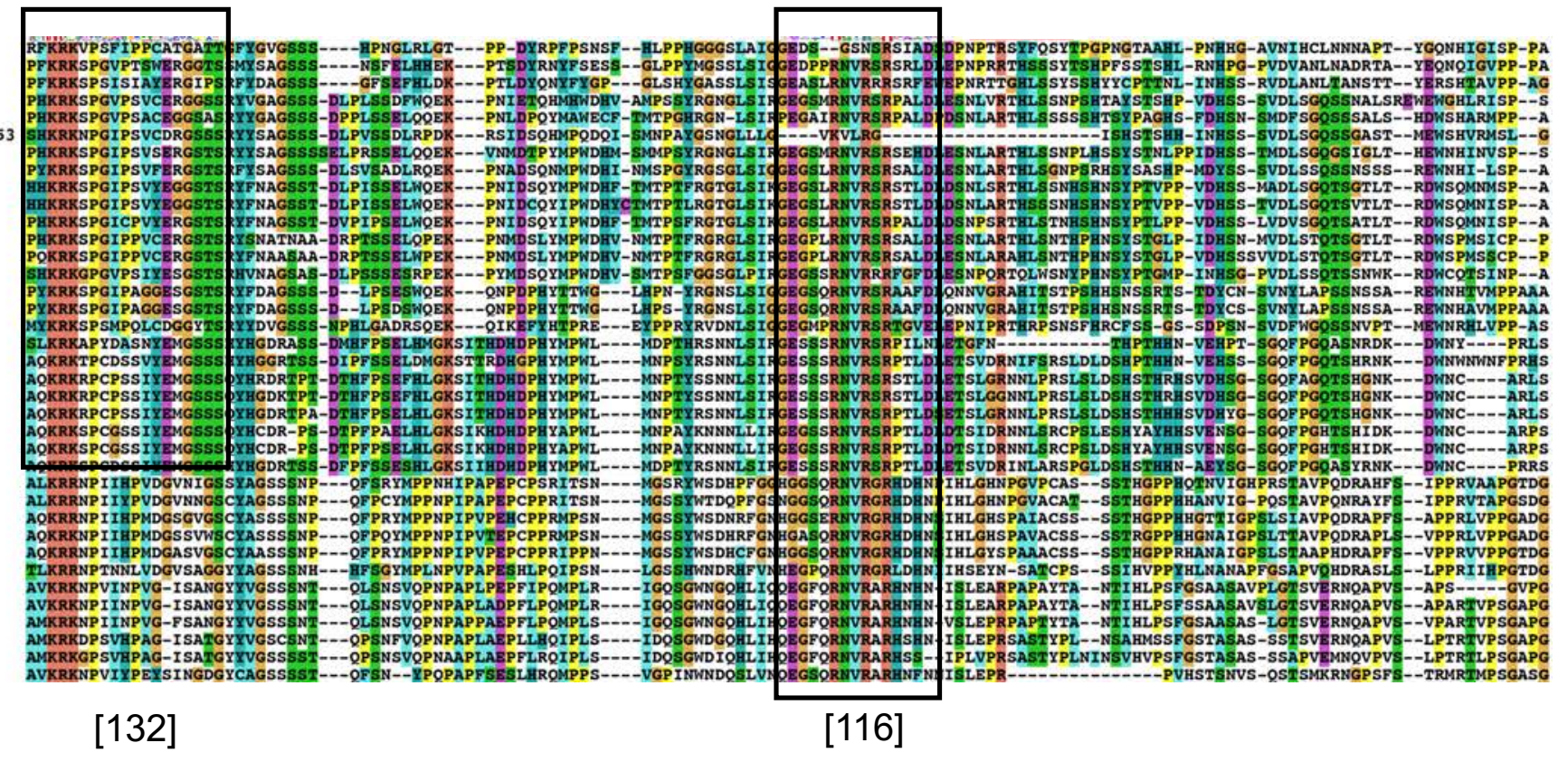




\section{Group D (cont.)}

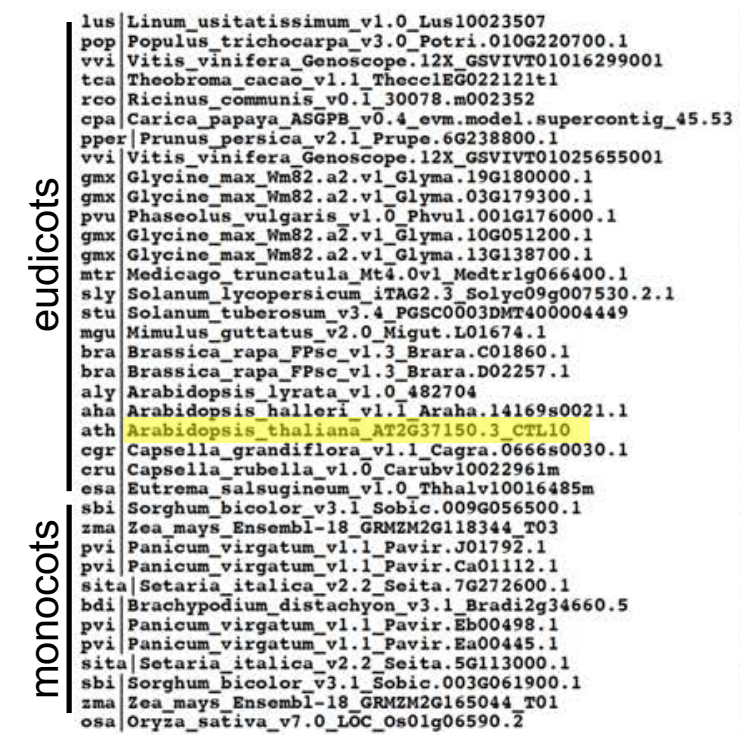

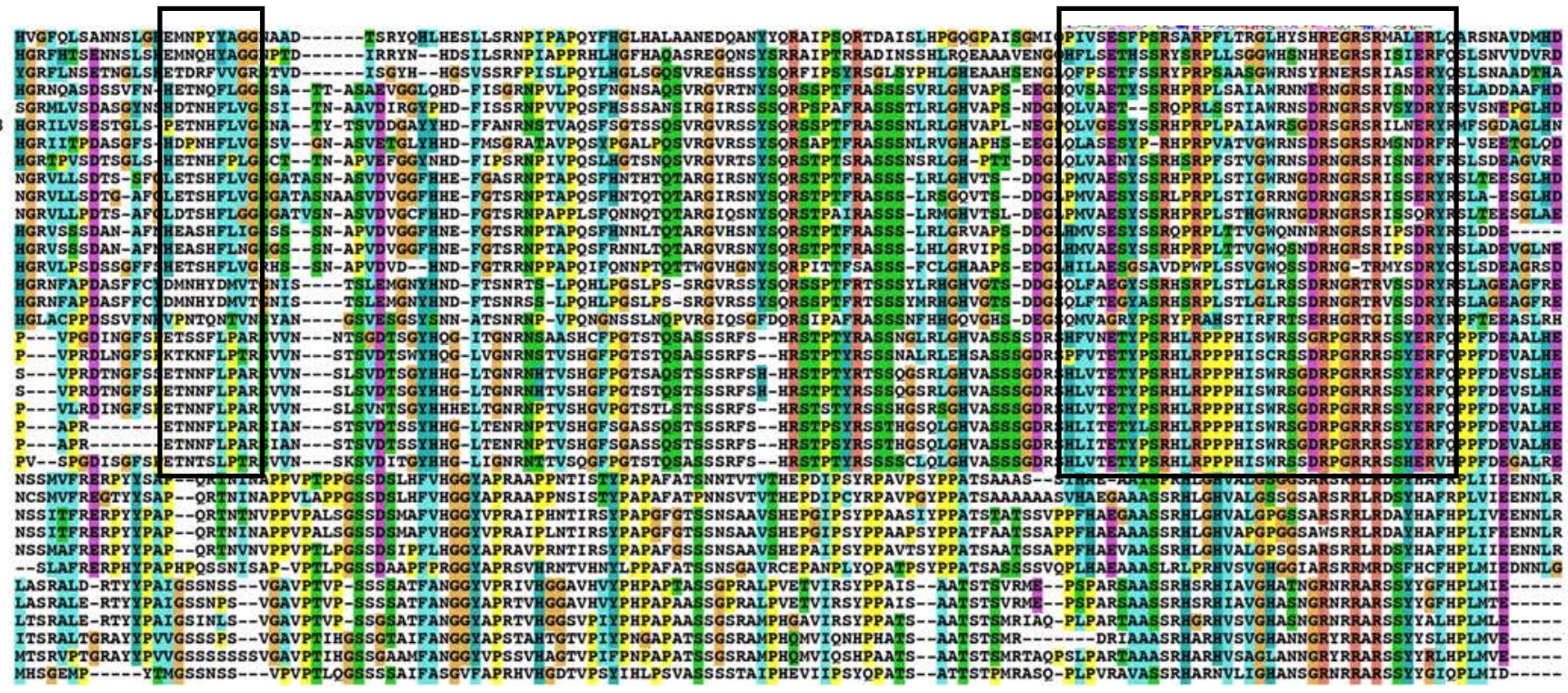

[144] 


\section{Group D (cont.)}

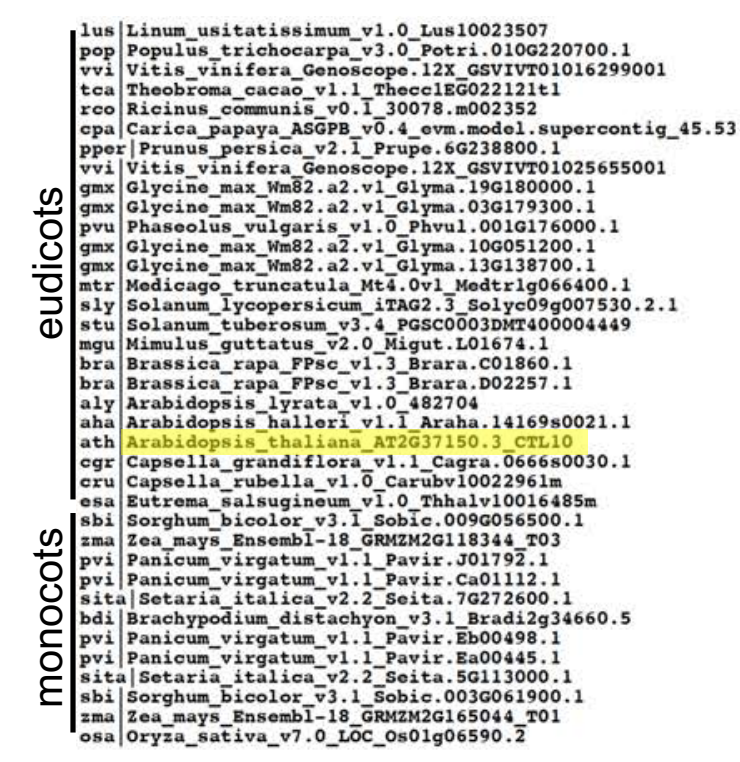

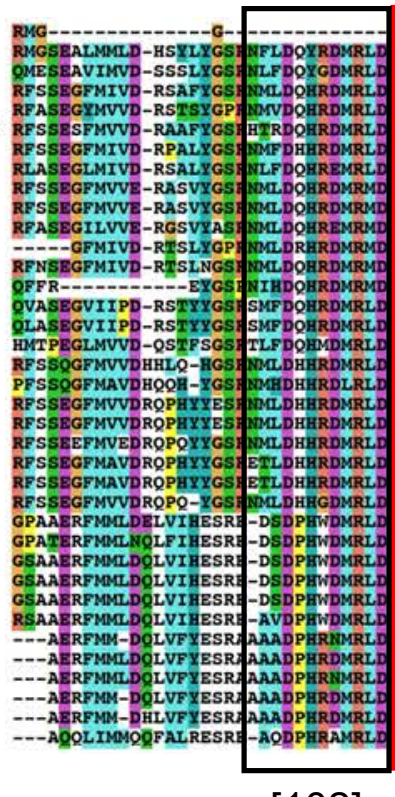

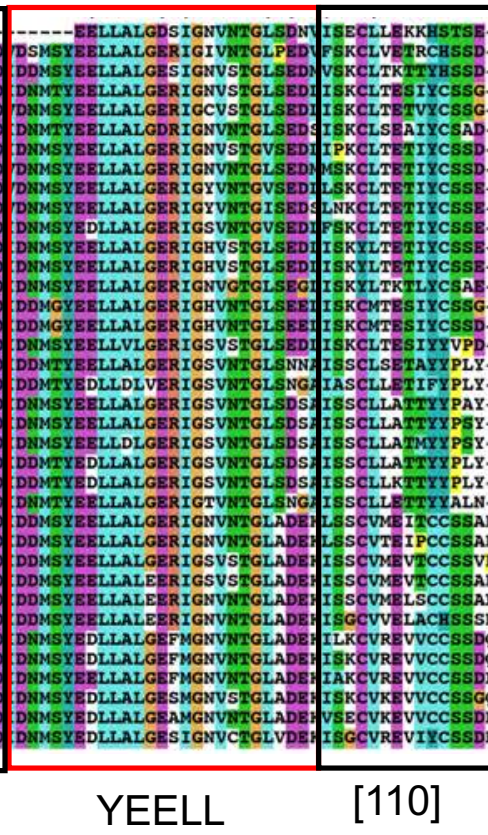

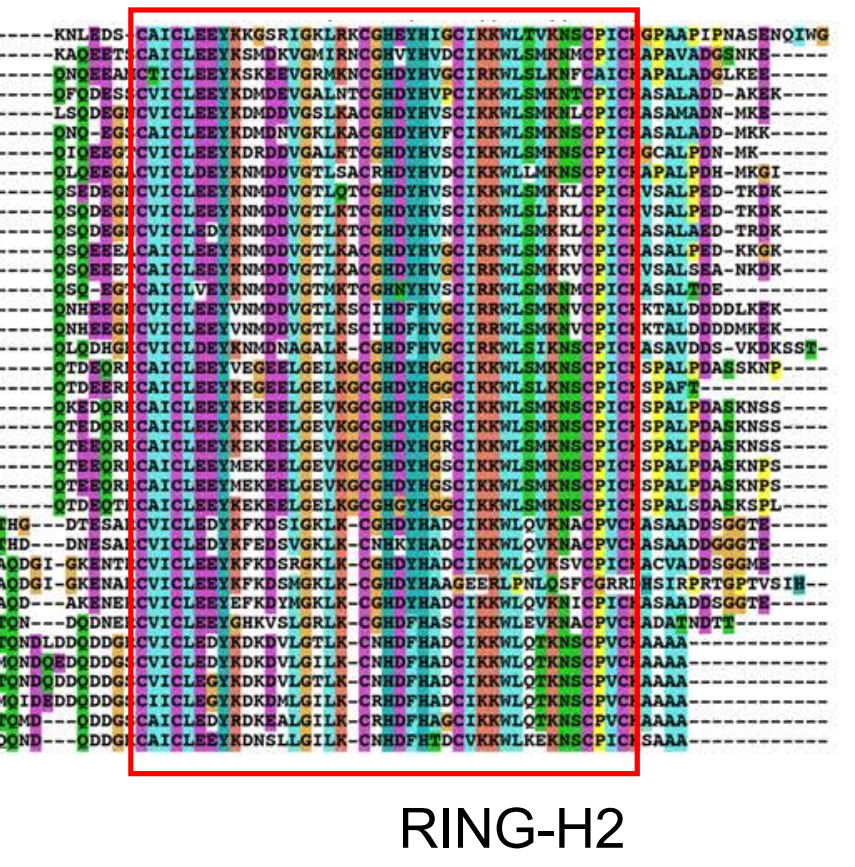




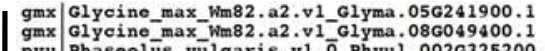
mpu Phaseoluss_vülgaris_v1.

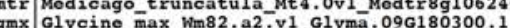
$\operatorname{mx}$ G1ycine-max_Wm82.22.v1-G1yma.07G097700.1 gr Eucalyptus_grandis_v2. pop Populus_trichocarpa - v3. 0 - Potri. 0126053800.1

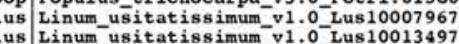
lus
lus Linum_usitatissimum_v1.0 Lus10013497
Linum_usitatissimum_v1.0_Lus 10021722 us Linum_usitatissimum Vitis_vinifora_Gonos zma zoa_mays Ensomb1-18. GRMZM26061663-Tro1 sbi Sorghum_bicolor_v3.1.Sobic.009621
pvi Panicum_virgatum_vi.1_Pavir.Ca01829.1 pvi
pvi sita|Setaria italica-v2.2-

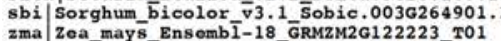
zma Zea_mays_Ensomb1-18_GRM2M2G122223_T01 bdi Brachypodium_distachyon_v3.1 Bradi2g47150.1 osa
ogrza sativa-v7.0_LC_oso1g49770.1
Capse11a_qrandifiora_v1.1_Cagra.0876s0008.1 ru Capsolla_rubolla_v1.0_Carubv1002049

th Arabidopsis thaliana ATIG73760,1 CTL14 ast
bst Boechera_stricta_v1.2.

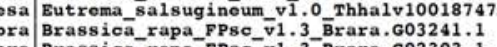
Gra Brassica_rapa-FPsc-v1.3.Brara.G02303.1 cru Capsella_rubella_v1.
bst Carubv10009526m
Boechera_stricta-v1.2-Bostr.7128s0404.

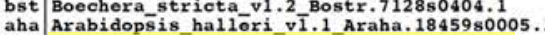
ath Arabidopsis thaliana_AT1G17970.1 CTL.15
bra Brassica_rapa_PPsc_v1.3_Brara.H02443.1 stu Solanum_tuberosum_- $\mathbf{v} 3.4$ P $\mathbf{P}$ GSC0003DMT40007853 aly Arabidops is 1yrata v $\overline{1}$. $03328 \overline{6} 0$

ath Arabidopsis thaliana AT5G67120.1 CTL11

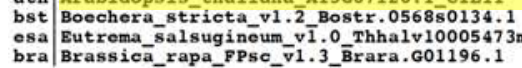

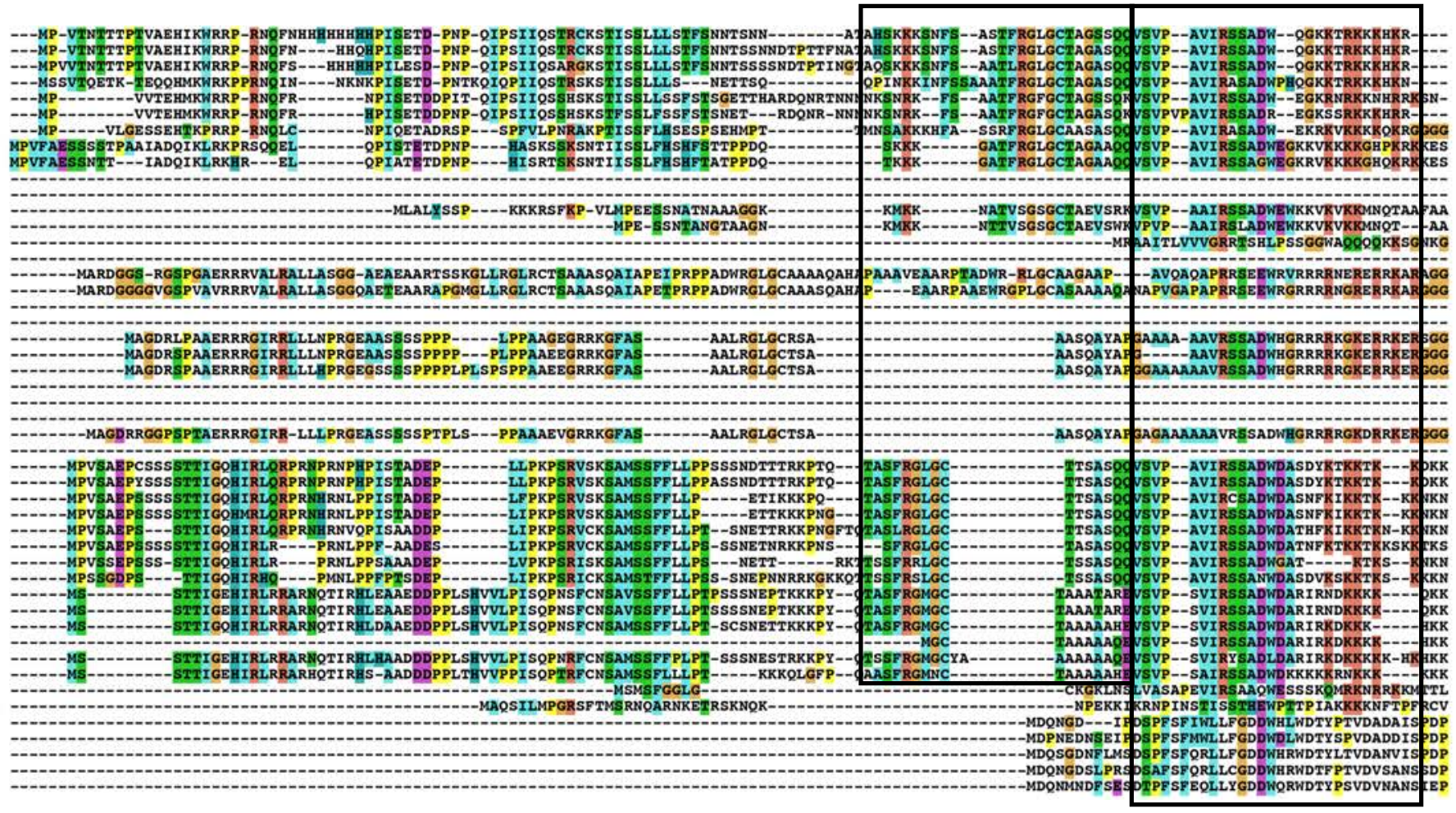

[134] 


\section{Group E (cont.)}

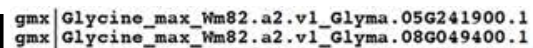
pvu Phaseolus_vulgaris_v1.0̄_Phvil.0026325200.1 max G1Ycino-max_hm82.a2.v1_G1 Yma. 096180300.1 gx Eucalyptus grandis_v2. ̄0 Eucgr. B00829.1 Pop Populus_trichocarpa v3. Do Potri.0126053800.1 us Linum_usitatissimum_v1. Lus L L L 10007967

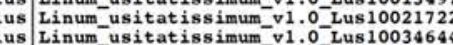
Vitis_-vinifera_Genoscopo.12X_GSVIVT01008343001 Vitis_vinifora_Genoscope.12X_GSVIVT0101 840001

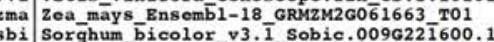
vi Panicum_virgatum_vi. 1 Pavir.Ca01829.1 vi Panicum_virgatum_v1.1_Pavir.Ea02502.1 sita|Sotaria_italica-v2:2-Soita.56280100.1 ma Zea_mays_EnsombI-18_GRMZM2G122223_T01

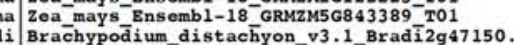
Oryza_sativa_v7.0_Loc_0s01g49770.1

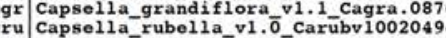

bidops is lyrata vi 10476516

th
bst
Boechera sa Eutroma_salsuginoum_vi 10 -Thhalv10018747 Brassica_rapa_FPsc-v1.3_Brara.G03241.1
bra
Brassica_rapa_FPso_v1.3_Brara.G02303.1 ggr Capsolla_granāiflō̄a_v1.1_Cagra.217580028.1 cru Capsella_rubella-v1.0. Carubv10009526m
bst Bochera_stricta_v1.2-Bostr.7128s0404. dopsis_halleri ví1.1 Araha.18459:

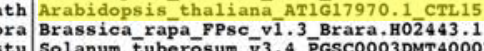

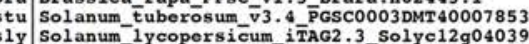
$1 \mathrm{y}$ Arabidopsis lyrata $\overline{\mathrm{v}} .0 \quad 332860$

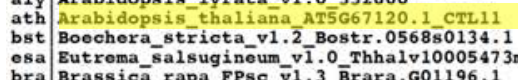

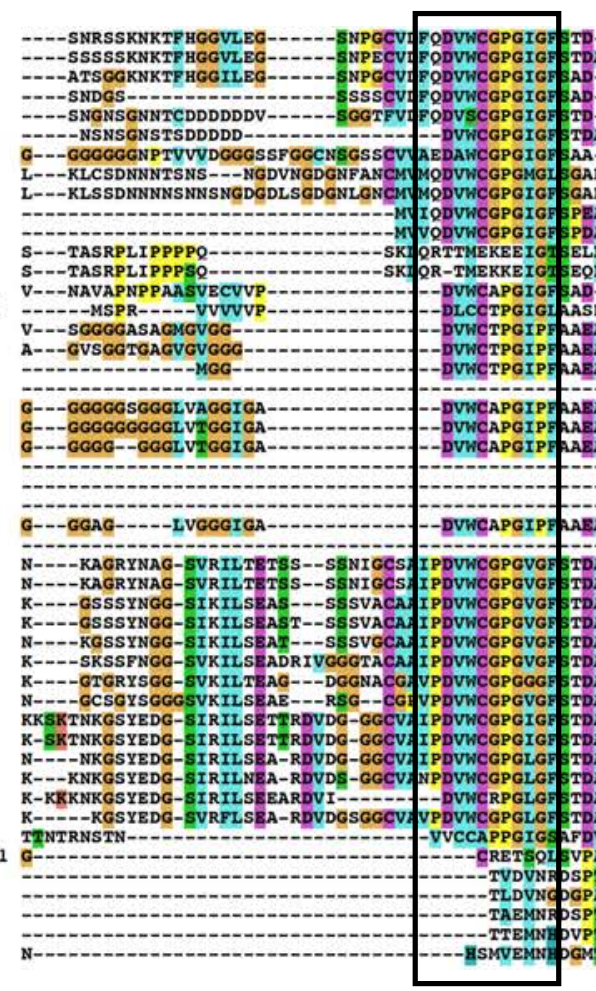

[118]

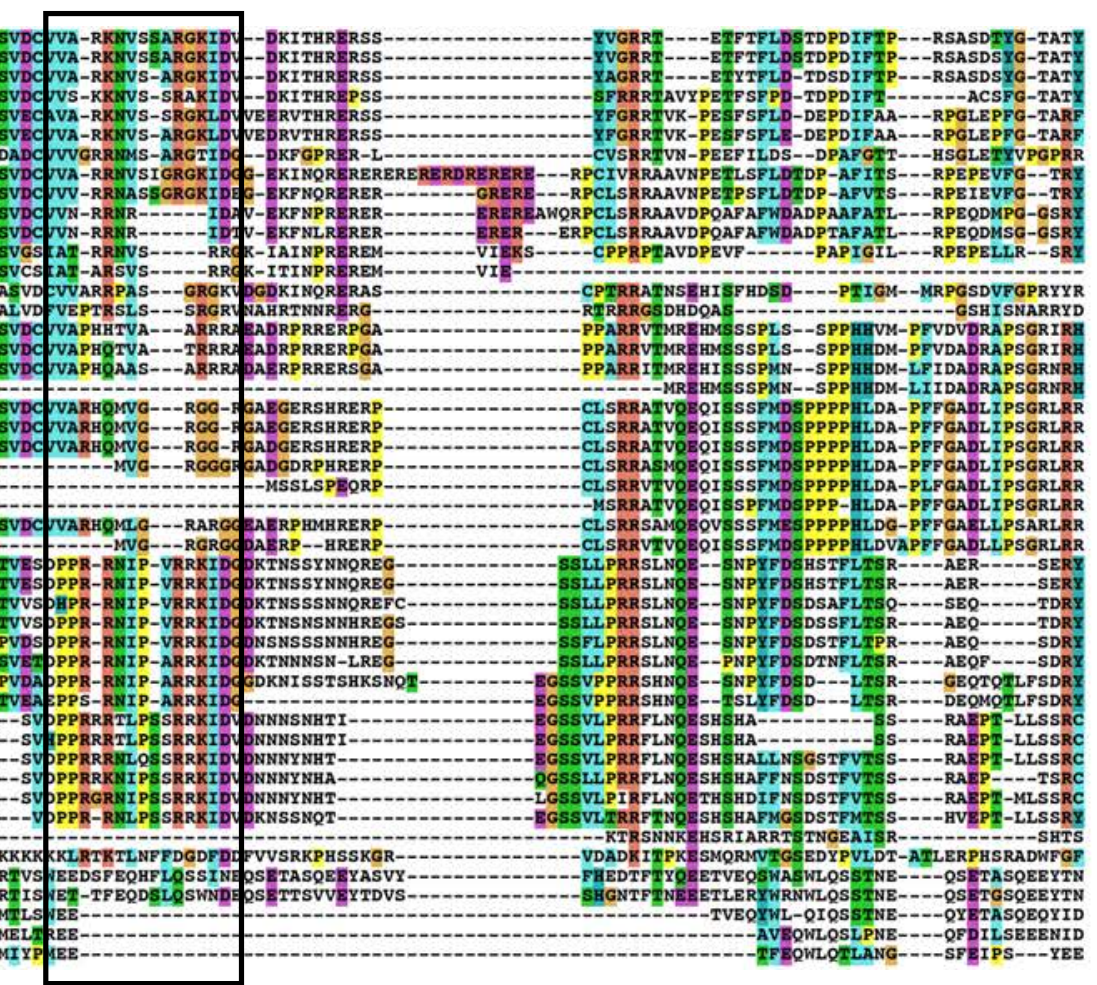

[116] 


\section{Group E (cont.)}

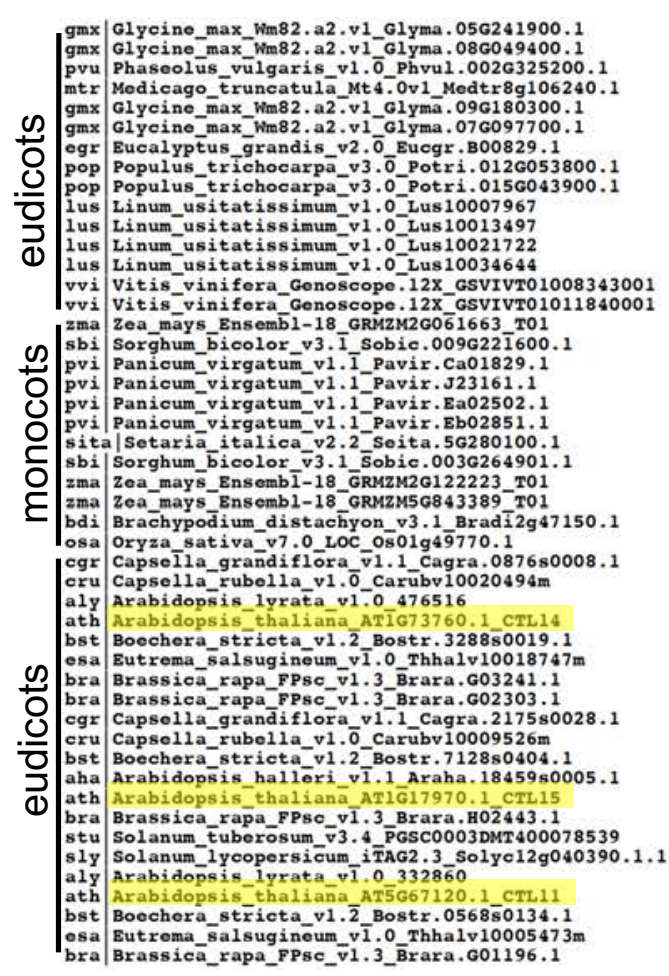

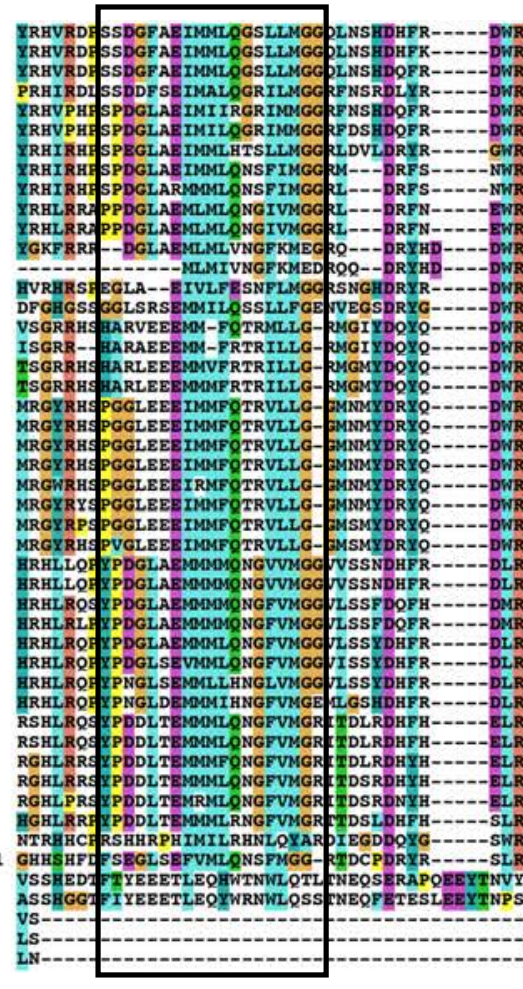

[125]

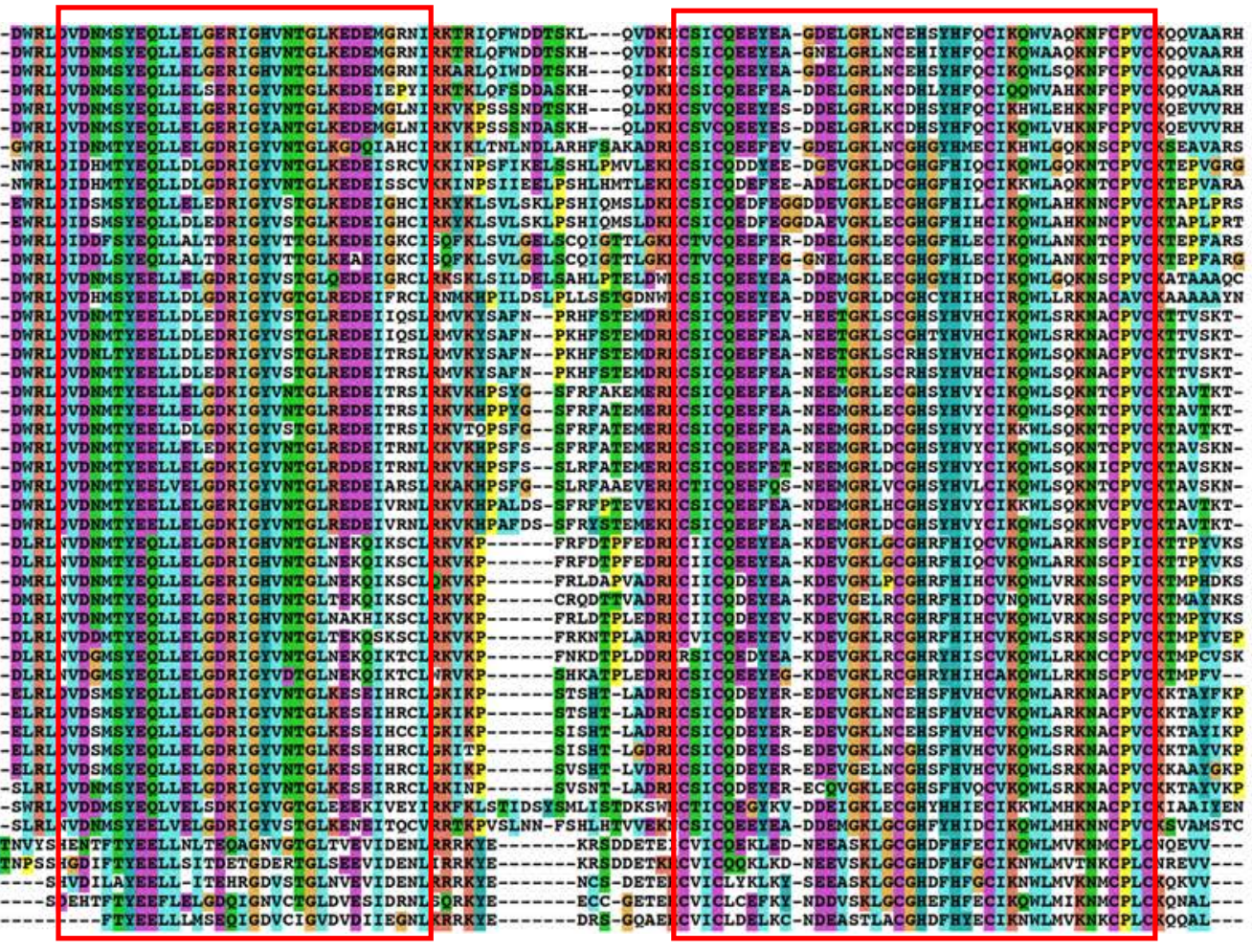

YEELL
RING-H2 


\section{Group F}

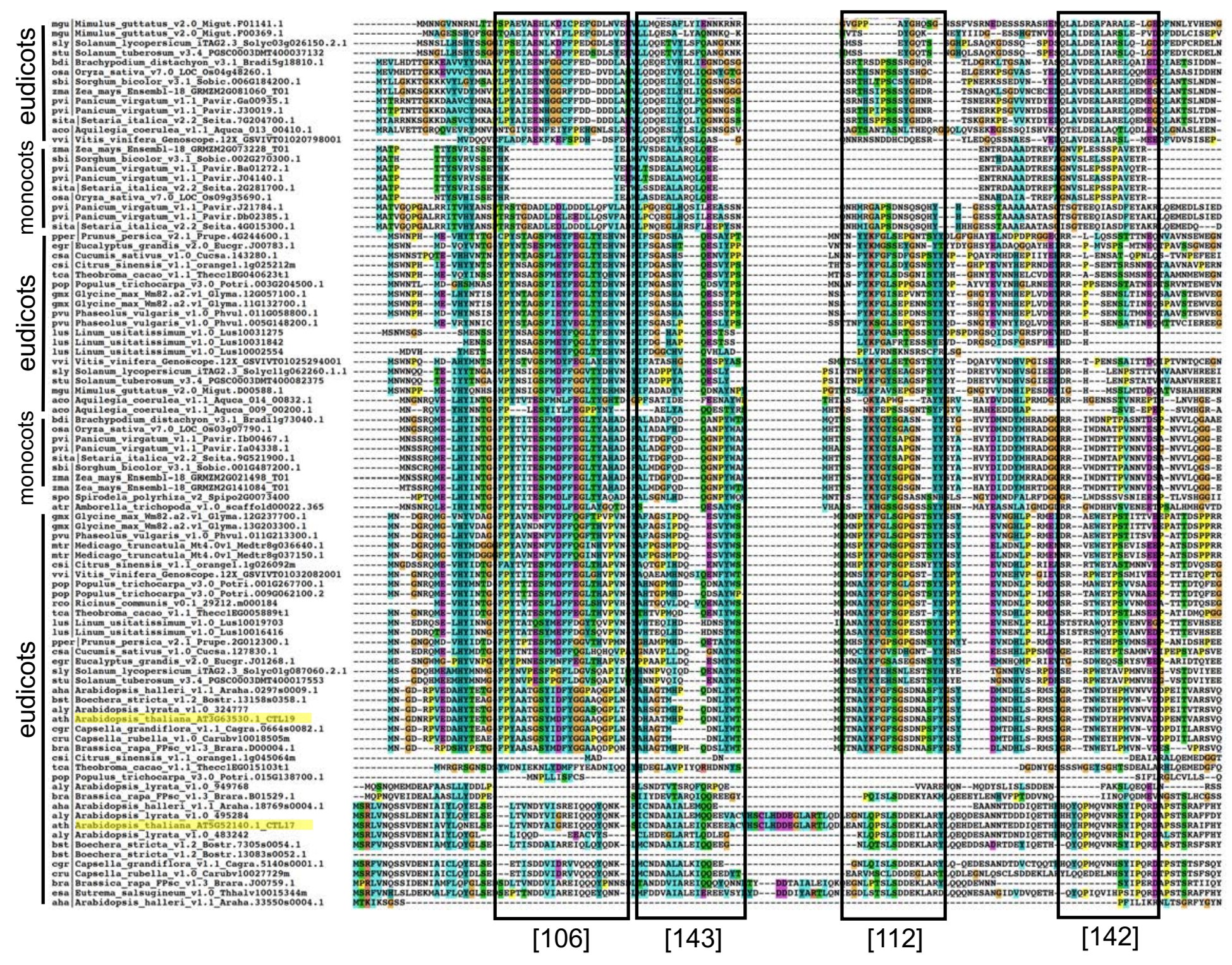




\section{Group F}

(cont.)

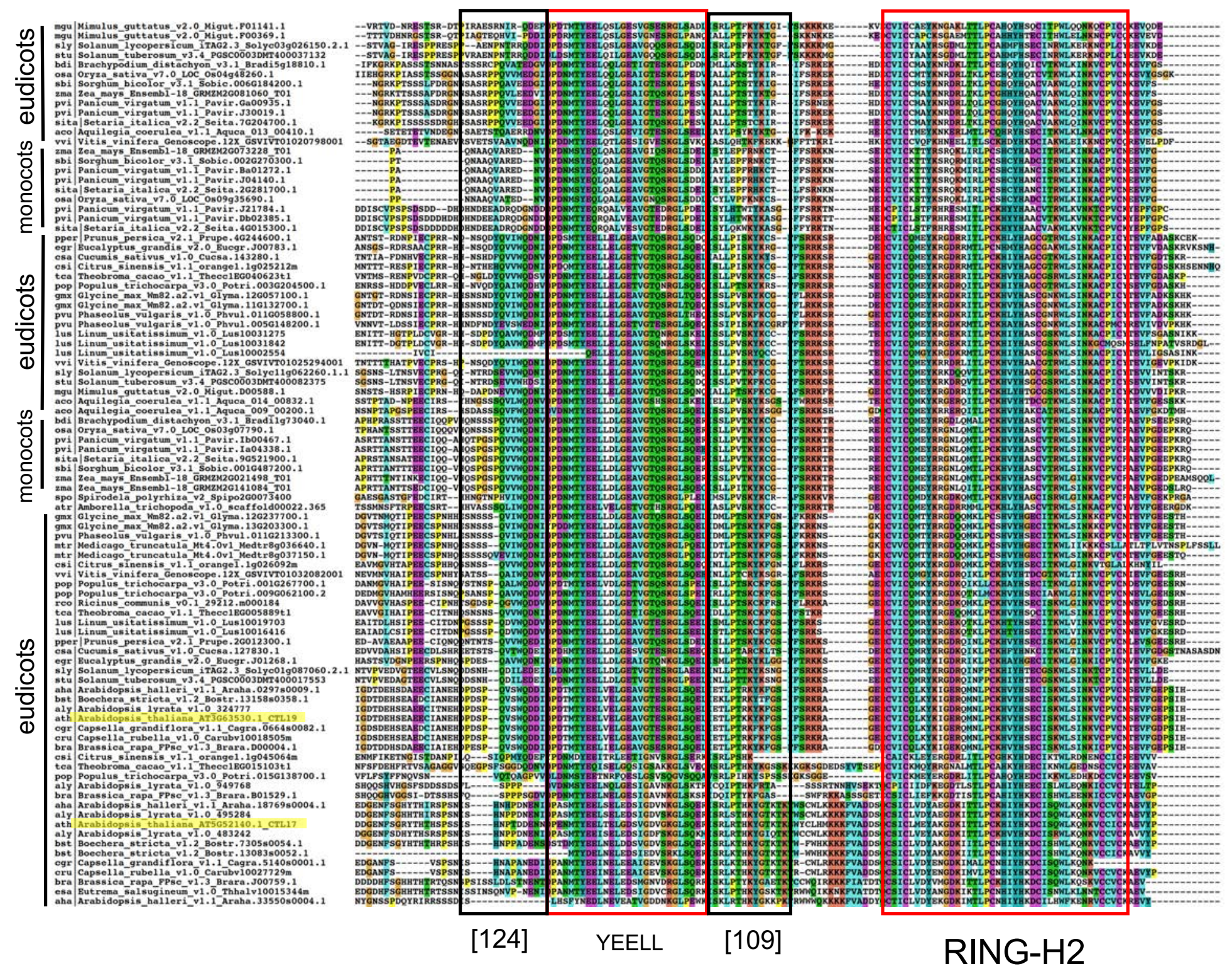




\section{Group G}

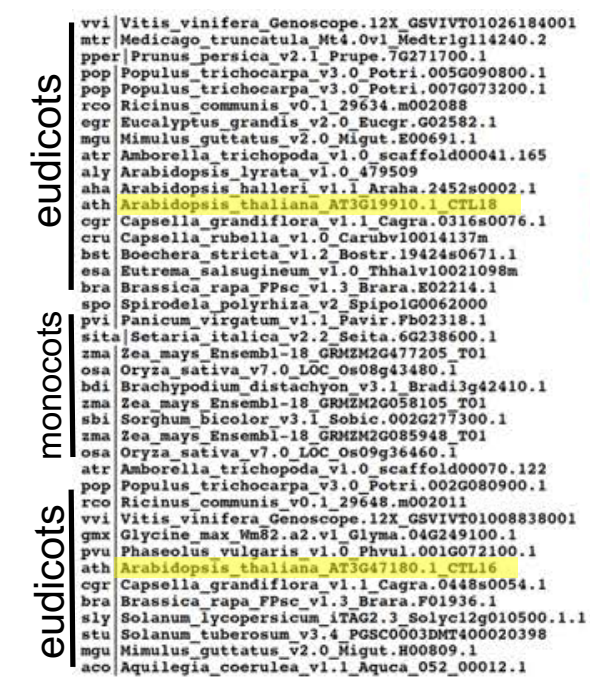

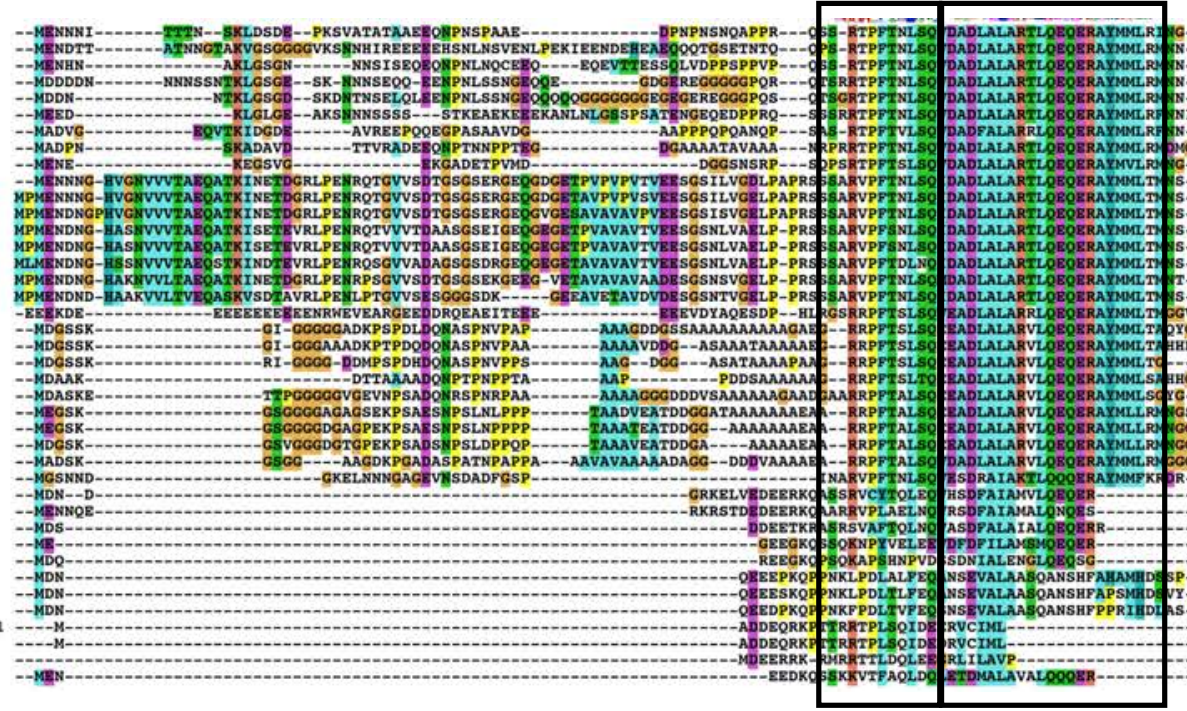

[137]

[103]

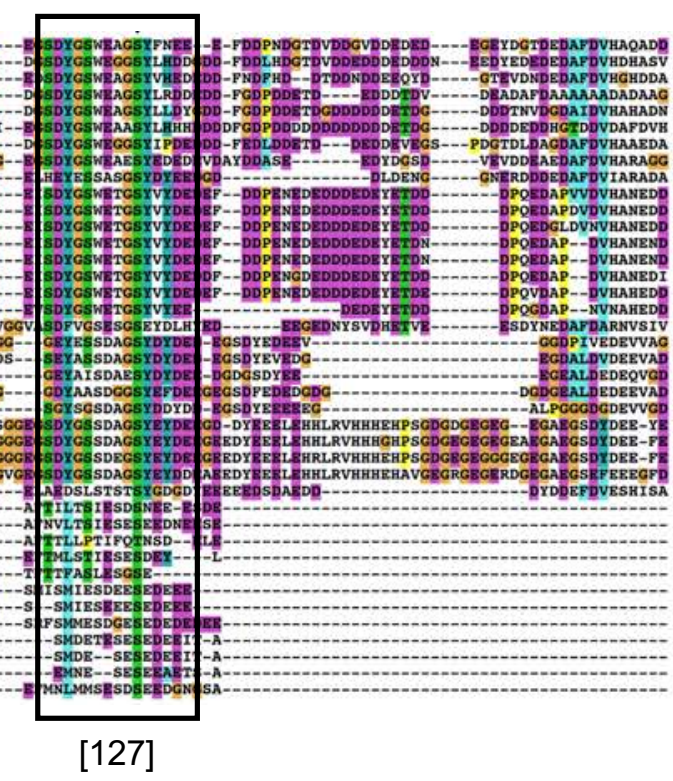




\section{Group G (cont.)}
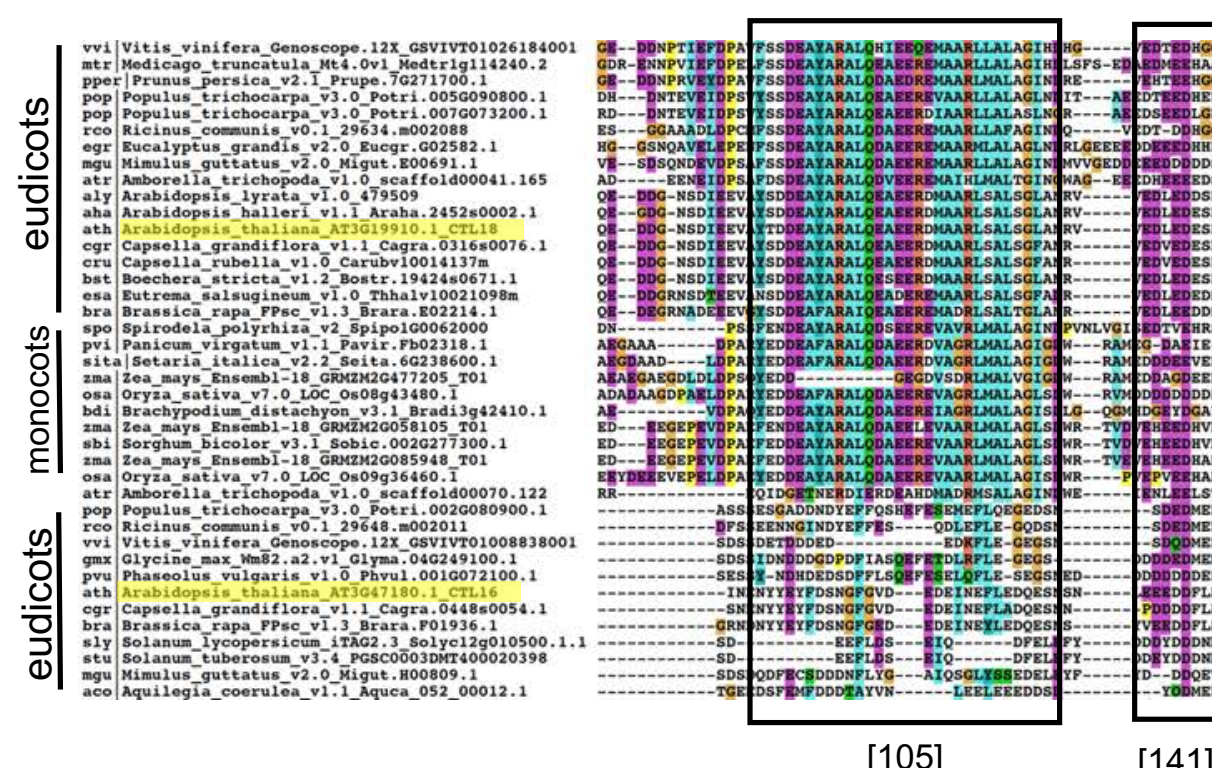

[105]

[141]
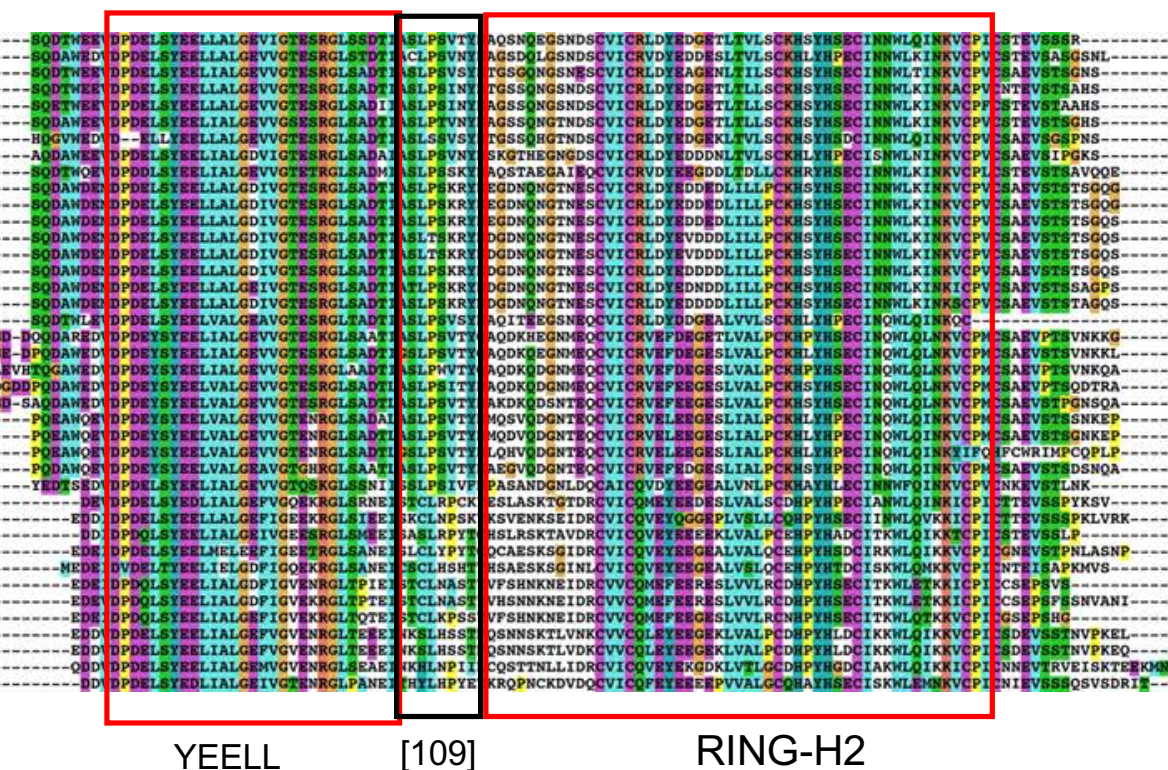

[109]

RING-H2 
